\title{
Modelling thrombosis in silico: frontiers, challenges, unresolved problems and milestones
}

Article

Accepted Version

Creative Commons: Attribution-Noncommercial-No Derivative Works 4.0

Belyaev, A. V., Dunster, J. L., Gibbins, J. M., Panteleev, M. A. and Volpert, V. (2018) Modelling thrombosis in silico: frontiers, challenges, unresolved problems and milestones. Physics of Life Reviews, 26-27. pp. 57-95. ISSN 1571-0645 doi: https://doi.org/10.1016/j.plrev.2018.02.005 Available at https://centaur.reading.ac.uk/75869/

It is advisable to refer to the publisher's version if you intend to cite from the work. See Guidance on citing.

To link to this article DOI: http://dx.doi.org/10.1016/j.plrev.2018.02.005

Publisher: Elsevier

All outputs in CentAUR are protected by Intellectual Property Rights law, including copyright law. Copyright and IPR is retained by the creators or other copyright holders. Terms and conditions for use of this material are defined in the End User Agreement.

www.reading.ac.uk/centaur

\section{CentAUR}


Central Archive at the University of Reading

Reading's research outputs online 


\title{
Modelling thrombosis in silico: frontiers, challenges, unresolved problems and milestones
}

\author{
A. V. Belyaev ${ }^{\mathrm{a}}$, J. L. Dunster ${ }^{\mathrm{b}}$, J. M. Gibbins ${ }^{\mathrm{b}}$, M. A. Panteleev ${ }^{\mathrm{a}, \mathrm{c}, \mathrm{d}}$, \\ V. Volpert ${ }^{\mathrm{e}, \mathrm{f}, \mathrm{g}, *}$ \\ ${ }^{a}$ M.V. Lomonosov Moscow State University, 119991 Moscow, Russia \\ ${ }^{b}$ Institute for Cardiovascular and Metabolic Research, School of Biological Sciences, \\ University of Reading, Berkshire, RG6 6AX, United Kingdom \\ ${ }^{c}$ National Medical Research Center of Pediatric Hematology, Oncology and Immunology \\ named after Dmitry Rogachev, 117997 Moscow, Russia \\ ${ }^{d}$ Center for Theoretical Problems of Physico-Chemical Pharmacology, Russian Academy of \\ Sciences, 119991, Moscow, Russia \\ ${ }^{e}$ Institut Camille Jordan, UMR 5208 CNRS, University Lyon 1, 69622 Villeurbanne, France \\ ${ }^{f}$ INRIA Team Dracula, INRIA Lyon La Doua, 69603 Villeurbanne, France \\ ${ }^{g}$ RUDN University, ul. Miklukho-Maklaya 6, Moscow, 117198, Russia
}

\begin{abstract}
Hemostasis is a complex physiological mechanism that functions to maintain vascular integrity under any conditions. Its primary components are blood platelets and a coagulation network that interact to form the hemostatic plug, a combination of cell aggregate and gelatinous fibrin clot that stops bleeding upon vascular injury. Disorders of hemostasis result in bleeding or thrombosis, and are the major immediate cause of mortality and morbidity in the world. Regulation of hemostasis and thrombosis is immensely complex, as it depends on blood cell adhesion and mechanics, hydrodynamics and mass transport of various species, huge signal transduction networks in platelets, as well as spatiotemporal regulation of the blood coagulation network. Mathematical and computational modelling has been increasingly used to gain insight into this complexity over the last 30 years, but the limitations of the existing models remain profound. Here we review state-of-the-art-methods for computational
\end{abstract}

\footnotetext{
* Corresponding author

Email addresses: aleksey_belyaev@yahoo.com (A. V. Belyaev), j.l.dunster@reading.ac.uk (J. L. Dunster), j.m.gibbins@reading.ac.uk (J. M. Gibbins), mapanteleev@yandex.ru (M. A. Panteleev), volpert@math.univ-lyon1.fr ( V. Volpert )
} 
modelling of thrombosis with the specific focus on the analysis of unresolved challenges. They include: a) fundamental issues related to physics of platelet aggregates and fibrin gels; b) computational challenges and limitations for solution of the models that combine cell adhesion, hydrodynamics and chemistry; c) biological mysteries and unknown parameters of processes; d) biophysical complexities of the spatiotemporal networks' regulation. Both relatively classical approaches and innovative computational techniques for their solution are considered; the subjects discussed with relation to thrombosis modelling include coarse-graining, continuum versus particle-based modelling, multiscale models, hybrid models, parameter estimation and others. Fundamental understanding gained from theoretical models are highlighted and a description of future prospects in the field and the nearest possible aims are given.

Keywords: arterial thrombosis, thrombin generation, computational systems biology, mathematical modelling

\section{Introduction}

Following injury, excessive blood loss is prevented by activation of hemostatic mechanisms that cause the blood to clot and platelets to aggregate, physiological processes that perform vital protective roles. These systems can also be

5 activated inappropriately, particularly as a result of vascular disease, leading to blood clot formation within the circulation, a dangerous thrombosis scenario that is the key trigger for heart attacks and strokes. The field of thrombosis and hemostasis represents a very special example of physical and chemical complexity of a living system, both because of its research challenges and (patho)physiological importance.

In order to maintain vascular integrity and stop blood loss, the systems of hemostasis are exquisitely complex. They incorporate two interdependent mechanisms, one mediated by blood cells known as platelets, and the second complex biochemical cascades that result in the conversion of blood from a liquid to a solid clot (coagulation). Platelet function encompasses processes of hy- 
dromechanics, cell adhesion and shape change, muscle-like platelet contraction, fluid-gel transformations together with intricate complexity of both intra- and extra-cellular biochemical reaction networks possessing complex spatiotemporal response dynamics. Coagulation is triggered by biochemical cascades involving numerous catalytic steps that culminate in the conversion of soluble fibrinogen into insoluble and polymerized fibrin. These processes influence each other, with coagulation pathways activated on the surface of activated platelets, and coagulation enzymes also activating platelets. Recent decades witnessed an explosive expansion of knowledge about this system, and yet its regulation (and even more deregulation) remains poorly understood. As a visible proof of our insufficient understanding, that disorders of hemostasis remain the leading immediate cause of death in the world. Various hemorrhagic and thrombotic complications are associated with numerous conditions ranging from sepsis to cancer, from stroke to myocardial infarction, from pregnancy to trauma, and play central roles in their pathophysiological development.

By definition, the way to understanding something complex is modelling, be it experimental, computational or mental models. Indeed, mathematical and computer models have been increasingly applied to solve biological problems in recent years. There has been an explosion in the number of papers on 35 "biomedical mathematical modelling", "computational systems biology", "virtual cell", "virtual human", "pharmacokinetic/pharmacodynamic models" and similar subjects. These papers abound with seductive phrases like "comprehensive model", "multiscale model", "fully three-dimensional model", etc. Computational biology is becoming immensely popular, although the majority of experimental biologists and medical doctors are still cautious and insufficiently familiar or comfortable with its use. In particular note, however, there has been considerable recent interest in the use of computational approaches to study thrombosis and hemostasis and an increase in the numbers, complexity and utility of associated computational models [1].

45

While computational studies provide important insights into different aspects of hemostasis and thrombosis, it is essential to understand that there are 
presently no models of these processes that could be described as comprehensive. The most successful models either focus on some aspects of hemostasis (e.g. blood coagulation in vitro or platelet adhesion), or use simplified laws to grasp general system dynamics. On the one hand, there are quite a few biological, physical or chemical things that we do not know about platelets [2, 3, 4, their formation into aggregates (thrombi) [5, 6] at sites of injury, or coagulation [7, 8]. At the same time, there are physical and mathematical constraints that make these models very hard to compute even when we do know the systems [9, 10]. For example, while 3-dimensional detailed models of adhering deformable blood cells are known, it would usually take weeks of supercomputer time to simulate several real-life seconds of platelet adhesion with a handful of platelets and red blood cells involved. As a result, models of thrombosis itself are rarely used in medicine or pharmacology. Testable predictions and fundamental hypotheses about thrombi are generated using simpler models [11, 10, 12, while mechanism-driven molecular-level insights are generated using models of signal transduction [13, 14, 15] or blood coagulation in vitro [1, 16], and not complex physiology-level models (with few exceptions) [17.

Why is it so? This review aims to answer this question, we shall discuss not successes but rather obstacles in the developing models of hemostasis and thrombosis. While we shall pay attention to progress in the field and to the promising developments (which are really exciting), the main focus will be the problems that should be overcome to develop models of thrombus formation for basic research and to aid clinical applications.

\subsection{Hemostasis and arterial thrombosis: from physics to physiology}

The state-of-the-art picture of a blood clot formation could be simplistically described as follows. Initially, right after injuring, the platelets adhere from the bloodstream to the injury forming a brickwork-like aggregate. At the same time, the plasma coagulates to produce fibrin and stiffen this aggregate. Thus a plug forms to prevent the blood leakage through the injury. There is a conventional terminological difference between two principal situations. If the blood clotting 
takes place near an open wound or a hole in a blood vessel, then the process is called "hemostasis". It is a positive, protective phenomenon. Yet sometimes the plug can grow inside a one-piece leakproof vessel due to numerous possible reasons (from inflammation to atherosclerotic plug rupture) - then it is referred to as "thrombosis". Both are thought to be the two sides of one coin.

In order to discuss challenges in the modelling of arterial thrombosis and hemostasis, we need first to describe its primary aspects, these are summarized in Fig. 1. There are two major physical mechanisms that stop bleeding upon vascular injury: platelet aggregation and fibrin clot formation. Platelets are specialized blood cells capable of adhering to the site of vascular damage and forming a plug to physically seal the wound. Fibrinogen is a protein present in blood at a substantial concentration (about $0.5 \% \mathrm{w} / \mathrm{v}$ ) and converted by action of thrombin into form fibrin following activation of the coagulation systems, which can form a three-dimensional network of fibers, which results in a local gel formation, thereby forming a physical clot. The critical steps in hemostasis and arterial thrombosis are shared, and this causes complications in the design of medications that are able to suppress thrombosis without adversely affecting hemostasis. Some reports do suggest processes, molecules or regulatory pathways whose effects are confined to hemostasis or thrombosis, whether these can be translated into safer therapies remain to be established, and consequently most anti-platelet and anti-coagulant drugs are associated with bleeding side effects. Amongst the complex and intertwined mechanisms of hemostasis and thrombosis, computational modelling holds the potential to better understand underlying mechanisms of action, and identify safer and more effective therapeutic targets.

The first critical step in the formation of either hemostatic plug or arterial thrombus is initial adhesion of platelets to collagen exposed at the site of vascular damage. In order to do this, platelets should come in contact with this collagen, and it is believed that interaction with red blood cells and red-bloodcell-mediated marginalization of platelets in the vessel [18, 19] are critically important for this 20, 21]. Therefore, the efficiency of platelet attachment is 
greatly dependent on rheology and the presence of erythrocytes 22 . Developing the correct micro-mechanical description of deformable cells in blood flow is regulation of coagulation by flow.

Fibrin formation is controlled by the blood coagulation network including 
about 50 proteins and co-factors interacting with each other as well as with blood and vascular cells. The core of this network is a cascade of proteolytic reactions with numerous positive and negative feedback loops. This cascade is a subject to complex spatiotemporal regulation, many elements of which are still unclear [26]. Fibrin allows solidification of hemostatic plugs or of the arterial thrombus. There are various interactions between blood coagulation and platelet-dependent hemostasis: for example, thrombin (the main enzyme of coagulation) is a major platelet activator.

Finally, there is the problem of limiting thrombus formation. Normally, after the thrombus was formed it stops its spatial propagation and is localized near the damaged site, so that the blood circulation can further take place in the organism. The physical and biological mechanisms behind this stoppage of thrombosis are not completely clarified (see Sections 3-5). As of now, there are numerous hypotheses about this [11, 10, 12, 27, 28, but there is less known about this than any other stage of platelet thrombosis.

\subsection{Biophysical and biochemical challenges for theory: things that we do not} know about thrombosis

There is a long list of uncertainties with regard to thrombosis/hemostasis mechanisms. Although some of them can be circumvented theoretically (e.g. by using phenomenological models), they remain a general impediment to the development of efficient models.

We dare to speculate that the mechanics of platelet-RBC interaction do not represent such a problem. The challenges in their correct representation are more computational as will be discussed in the respective section.

VWf action and interaction with platelets and collagen (or other substrates) is, however, different. While there is some understanding of its mechanics, it is far from complete. The ability of this protein to interact with different platelet receptors, its ability to be influenced by flow conditions, combined with its ability to support platelet aggregates make it interesting and difficult to model.

The stages of platelet aggregate formation and blood coagulation are even 
Activators:

collagen, thrombin, ADP, TxA2, etc.
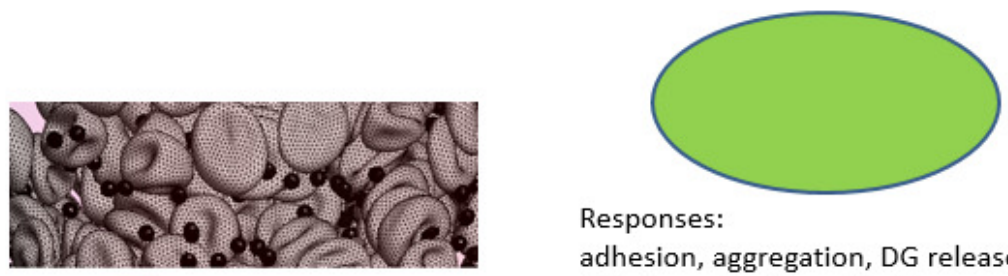

Responses:

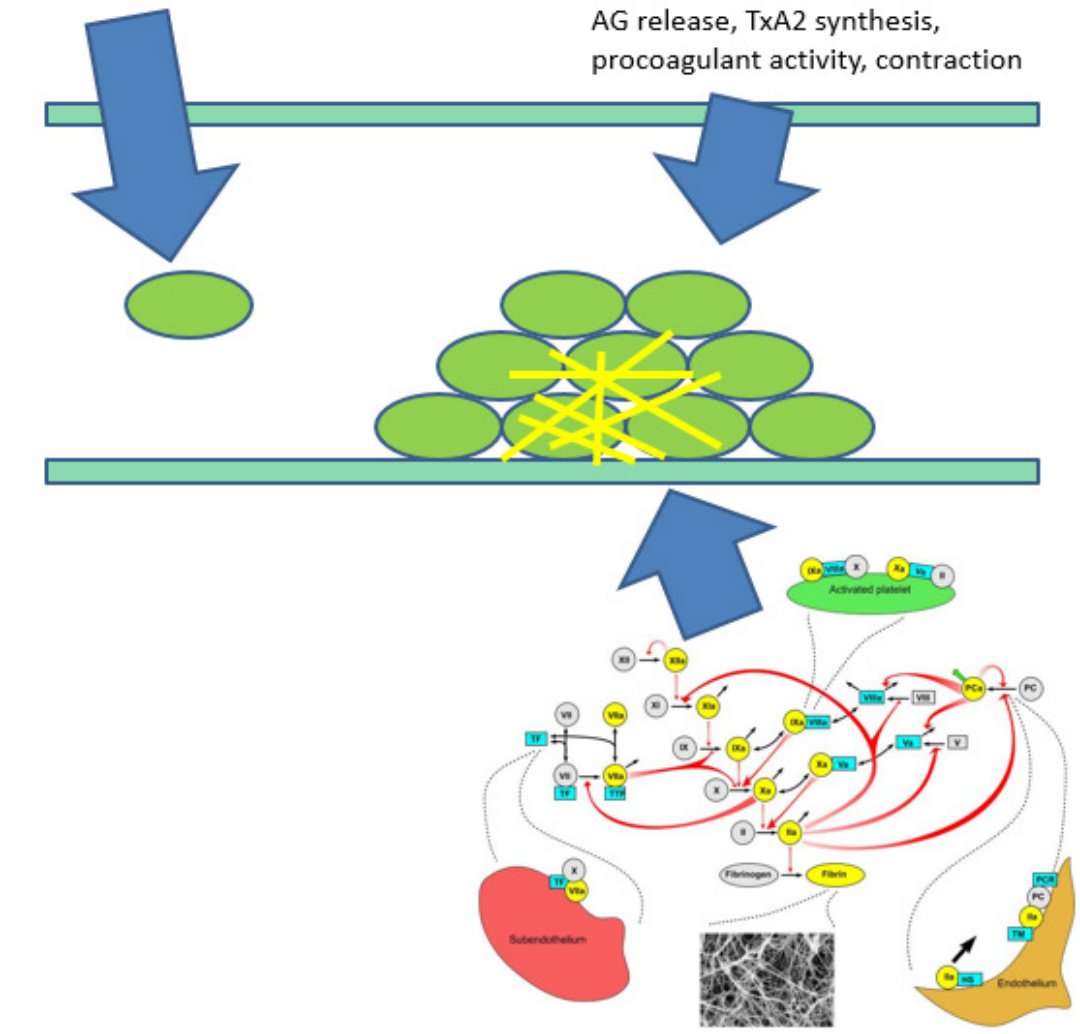

adhesion, aggregation, DG release,

AG release, TxA2 synthesis,

procoagulant activity, contraction

Figure 1: Schematic representation of blood coagulation process. Blood flow with erythrocytes and platelets (upper left, reprinted with permission from [19] ) and platelet with its role in clotting (upper right). Coagulation cascade (lower image).

more puzzling. Here is a brief list of subjects that remain open and challenging

in the field. It should be clearly emphasized though, that these open subject 
are not exactly obstacles for theoretical research, but rather opportunities to apply computational methods for the solution of interesting problems. They are, however, obstacles for medical applications of computer models because a model for clinical or pharmacological application should be relatively free of such uncertainties.

1. The role of the contact pathway in arterial thrombosis (but, supposedly, not in hemostasis). The coagulation network (see Fig. 1) can be activated in two ways. It can be initiated by contact of a protein, called factor VIIa, with a cofactor, the transmembrane protein tissue factor, at a site of vascular damage. This so-called extrinsic pathway is believed to be the main way of coagulation activation in hemostasis. Alternatively, there is a protein called factor XII at top of the coagulation cascade that can be self-activated via contact with foreign materials. This "contact pathway" is the main way of clotting activation in vitro, but deficiency of factor XII is not associated with bleeding tendency. The contact pathway was not a source of interest to researchers until this last decade, when it was found to be potentially involved in arterial thrombosis [29]. This could be one of those rare cases when a biochemical reaction plays different roles in thrombosis and hemostasis, and we still do not know why.

2. The effects of platelet-derived polyphosphates and coagulation activation by platelets. A related issue is the ability of platelets to not only accelerate coagulation reactions but even initiate coagulation. This supposedly occurs via release of polyphosphate from dense granules and contact pathway [30], though there is evidence by different groups, including ours, that other mechanisms could be involved [31.

3. The functions of procoagulant platelets. One of the most mysterious questions in the field is why activated platelets form at least two subtypes: "aggregating" platelets that can actually form the body of a hemostatic plug or thrombus and procoagulant platelets that can accelerate reactions of blood coagulation [32, 33, 34, 6]. As of now, there is a lot of information 
about signalling pathways leading to these platelet subpopulations and their properties, but no real idea about their physiological or pathological significance.

4. The role of neutrophils in platelet adhesion during thrombus formation. A role of other blood cells types, most notably neutrophils, in formation of various types of thrombi is being increasingly recognized. They are likely to contribute both mechanically (in the adhesion) and biochemically, and this is one of the subject that is lacking in all the existing computational models.

5. Cell-derived microparticles and their numerous roles. Another component of blood with interesting and unclear abilities are cell-derived microparticles. These lipid bubbles, ranging in size from tens of nanometers to more than a micron, possess a number of hemostasis/thrombosis-related activities including ability to both activate and propagate coagulation [35, 36, 37. These include the presence of adhesion receptors, tissue factor, phosphatidylserine and others. Some microparticles are present in circulation and are greatly increased in number in case of inflammation or cancer, while others are produced during thrombus formation as a result of platelet activation. While they are recognized as a prothrombotic marker, their roles in hemostasis and thrombosis are unclear.

6. The unique mechanical properties of fibrin. Finally fibrin gels themselves, neither really fluid nor solid, represent an interesting challenge for computer modelling [38. It is probably les important for arterial thrombosis (mainly driven by platelet accumulation) but is critical for venous one.

1.3. Thrombin generation and spatiotemporal regulation of blood clotting: a problem in itself

The blood coagulation network as depicted in Fig. 1 is a very interesting example of a biological network with well-known biochemistry but poorly understood regulation. Although the main reactions of blood coagulation were discovered over 20 years ago and there have been many models of coagulation 
published [39, 40, 41, 42, 43, 44] there remains no model that can be successfully used alongside experiments with it having to be individually tailored and adjusted. This is in spite of some models being compared to experiments, e.g. our own model [4] that has been employed for data analysis in a number of studies [25, 39, 45, 46]).

The term "thrombin generation" has two meanings: it describes both a) formation of the main clotting enzyme thrombin during blood coagulation under any conditions, and b) the so-called thrombin generation assay, where thrombin concentration in plasma is determined as a function of time in vitro following stimulation in a controlled setting [47, 48]. While any computer model of blood coagulation necessarily includes thrombin generation in the first meaning, here we shall focus on the second one, because wide use of the thrombin generation assay for basic research and clinical data necessitated development of computer models for the data analysis and at the same time provided a lot of information for model validation. The typical design of the assay includes use of 96-well plates, where samples of plasma are recalcified, supplemented with phospholipid, tissue factor and a fluorogenic substrate cleavable by thrombin and able to indicate thrombin concentration.

There are a number of interesting challenges that are specific for thrombin generation.

1. How the onset of coagulation is regulated? The physiological activator of coagulation, tissue factor, is not inactivated by itself. However, once it forms a complex with factor VIIa, it can be inhibited by TFPI (Tissue Factor Pathway Inhibitor). This Kunitz-type inhibitor has a beautiful negative feedback mode of action: one of its Kunitz domains can bind factor Xa (that is produced by VIIa in complex with TF), and then another domain can bind the VIIa-TF complex. The problem is that this two-step inhibitory mechanism can not describe experimental data of thrombin generation, with and without TFPI [7. There are hypotheses to explain this discrepancy, but the truth is that we do not know how the first step 
of coagulation is really regulated.

2. Background contact activation. All real-life experiments take place in tubes, plate or other labware. As foreign surfaces, they activate coagulation via the above-described contact pathway [49]. This contribution depends on the experimental design, but in many cases can significantly affect the results unless a specific inhibitor, like corn trypsin inhibitor or something else, is used [50]. Most clinical protocols of thrombin generation involve significant contributions of contact activation.

3. Membrane-dependent reactions of blood coagulation. The majority of blood coagulation reaction occur not in solution, but on the surface of membranes provided by platelets, endothelial or sub-endothelial cells. These reactions harbor quite a number of surprises [33, 34, 51, and their description in the existing model is but a first-order approximation that is applicable within a narrow range of conditions.

4. Other. There are a number of other problems that include factor XI activation by thrombin [52] (a record-breaking reaction with 5-order-ofmagnitude difference in the experimentally reported rates unless we count those who do not believe it to occur at all), problems with protein $\mathrm{S}$ (infamously irreproducibly active), and a number of recently discovered reactions.

Once again, it should be stressed that these mysteries do not prevent researchers from developing models for the purposes of research. In contrast, such models can be used to get insight into these problems. However, in order for a model to be applied in clinical situations, it has first to be established in its basic features, and this is what is made difficult by our insufficient knowledge.

In the following sections we will discuss blood coagulation and clot growth beginning with thrombin generation (Section 2). We will introduce spatial effects (Section 3), fluid motion (Section 4) and the influence of blood cells (Sections 5 and 6 ). Finally we conclude with perspectives and future challenges. 


\section{Thrombin generation}

The primary function of the coagulation system is the termination of bleeding caused by vessel wall damage. This is achieved by covering the injured site with a fibrin clot that appears at the final stage of a complex proteolytic enzymatic cascade where activated clotting factors act as catalysts for activation of others [53, 54]. The key enzyme of this coagulation cascade is thrombin (Figure 1), it uniquely catalyses the conversion of fibrinogen to fibrin as well as playing a crucial role in controlling the kinetics of clot formation [53, 55, 56]. Thrombin formation can be launched by tissue damage that ruptures the endothelium exposing tissue factor to blood flow (this is called the extrinsic pathway), or by contact with a foreign substance where activation of factor XII triggers activation of factor XI (this is called the intrinsic pathway) [54, 57, 58]. Both pathways lead to the activation of factor $\mathrm{X}$ that contributes to the conversion of prothrombin to thrombin [54]. Once thrombin concentration reaches a threshold value, further prothrombin activation takes place due to positive feedback loops within the coagulation cascade [54, 57, 40].

There are numerous works devoted to modelling the coagulation cascade under different simplifying assumptions, these are necessary since the complete reaction scheme is complex and not completely known, especially if platelets are involved. In vitro coagulation kinetics through the extrinsic pathway were modelled in [59, 60, 61, 62, 63, and through the intrinsic pathway in [64, 65, 66. 67. All of these models exclude platelets, their influence being taken into account in [68, 69, 70, 71]. In vivo coagulation kinetics is studied in [72, 73, 74, 75, 76, 77. These models all vary in the number of reactions (from 12 to 93), concentrations (from 6 to 92) and parameters (from 16 to 48) included, reflecting the simplifying assumptions underpinning their construction.

The coagulation cascade is often characterized by thrombin generation curves (TGC) that represent the variation in thrombin concentration over time. Typical TGCs in response to various concentrations of tissue factor (extrinsic pathway) are shown in Figure 2. These are characterized by their lag time (beginning 
thrombin potential (ETP, the area under the curve) and start tail time (concentration close to 0 ). These values depend on the initial concentrations of blood factors and on the reaction rates. Increases in the initial concentration of tissue factor decrease the lag time and time to peak but increase maximal thrombin the opposite effect, as occurs in blood from haemophiliacs.

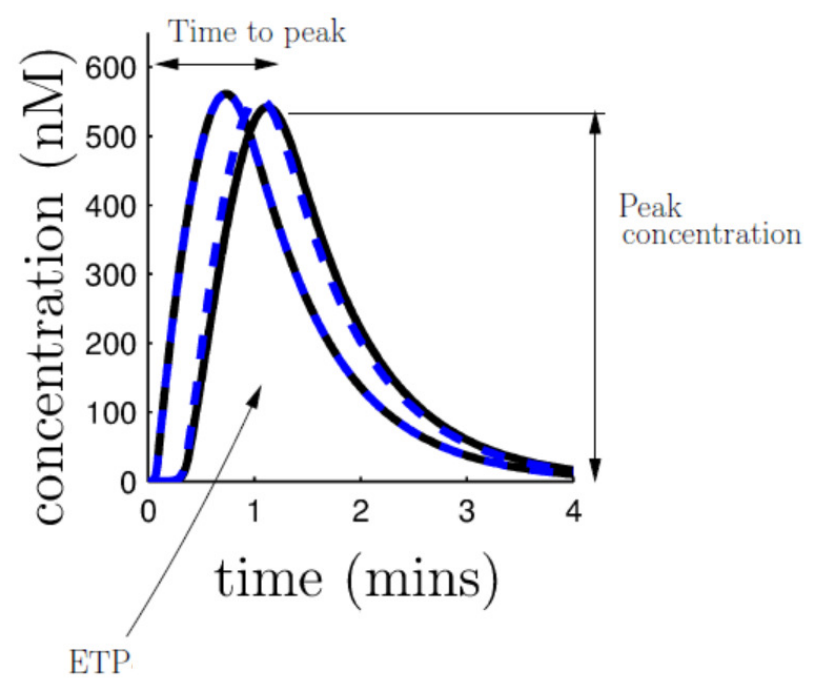

Figure 2: Asymptotic approximations (broken lines) are able to capture thrombin concentration growth and its decay (equations 11, 41), these are shown against numerical simulations of the full model [78] for various levels of stimulant (parameter $k_{1 a}$ ). The asymptotic analysis is also able to provide simple equations that express the key metrics used to describe TGCs (time to peak, peak thrombin concentration and ETP) in terms of initial conditions and reaction rates.

Thrombin generation is not a perfectly standardized assay: differences in the protocol and in the equipment could produce a large range of discrepancies between the laboratories $[79,80$. All the models cited above can fit individual 330 curves, given a proper choice of parameters, and TGCs can even be described by a simple combination of exponential functions with four parameters [81, 82]. 
However, there are numerous difficulties with the justification of these results and their clinical applications:

- Recent studies show that these models with their "original" values of parameters give results that are very different from clinical data on healthy and hemophilic subjects 83]. Calibration (fitting) of the parameters improve the results but the errors remain large (mainly in the interval $30-50 \%$ for the ETP and 50-200\% for the maximal value).

- It is not clear whether theoretical models can be used to predict the dependence of TGCs on the initial concentrations of blood factors. Analysis of results shows that the precision of the theoretical curves decreases when varying these concentrations 84. This question has a crucial importance for the treatment of blood coagulation disorders and of various diseases that influence blood coagulation (cancer, HIV and so on).

345 - There are a large variety of models proposed, describing alternative experimental or clinical conditions, and it is not clear whether these are valid under alternative conditions. More complete models (with more variables and parameters) are not necessarily more precise because of the uncertainty with the parameters and individual variability. of thrombus formation. The development of a multiscale model of thrombus formation necessarily involves multiple molecular and cellular components that bridge both length scales, that vary from nanometers through to centimeters (i.e. size of a protein to the diameter of a blood vessel), and time scales that vary from milliseconds through to hours (i.e. protein reactions through to vascular remodeling). The incorporation of the coagulation cascade and thrombin generation into a model of thrombus formation can highlight the mathematical and computational challenges in formulating and analysing models of such complex and disparate systems. This involves the development of models at each scale as well as their integration into a cohesive whole. The challenge is that if all the details of a model developed at a finer microscale (such as thrombin 
generation) are incorporated into a macroscale model (such as thrombus formation) then the complexity, and alongside it the computational costs, become problematic. To overcome this challenge it is necessary to reduce the complexity at the finer scale while retaining the models important dominant behaviour, that can they be passed to the model at the coarser scale. One method that can be used to bridge time and/or space scales in this manner is matched asymptotic expansions. A demonstration of this is given in 41] where the complexity of the extrinsic pathway described in the full model 78 (cf. Figure 1) is reduced to a smaller approximation that captures the dominant reactions behind the growth and inhibition of thrombin:

$$
\begin{aligned}
\frac{d P}{d t} & =\frac{f(C) P}{P+k_{1}}, \quad \frac{d T}{d t}=\frac{f(C) P}{P+k_{1}}-k_{2} T, \\
\frac{d V_{5}}{d t} & =k_{3} T+\frac{k_{4} V_{10}}{k_{5}+P}-k_{6} V_{5} V_{10}, \\
\frac{d V_{10}}{d t} & =k_{7} e^{-\gamma t}-k_{6} V_{5} V_{10}, \quad \frac{d C}{d t}=k_{6} V_{5} V_{10},
\end{aligned}
$$

where $P$ is the concentration of prothrombin, $T$ the concentration of thrombin, $V_{5}$ and $V_{10}$ are the concentrations of activated factors $\mathrm{V}$ and $\mathrm{X}$, respectively, $C$ is the concentration of prothombinase complex, and $f(C)=k_{9}+C-$ $375 \sqrt{\left(k_{9}+C\right)^{2}-k_{10} C}$. The term $k_{7} e^{-\gamma t}$ describes the activation of factor $\mathrm{X}$ at the damaged vessel wall.

This reduced system of equations 1 is able to provide an excellent substitute for the full model, this is demonstrated in Figure2, It correctly describes the key TGC characteristics, such as peak thrombin concentration, in terms of initial conditions and reaction rates.

Figure 1 demonstrates how such reduced model obtained via the method of matched asymptotic approximations may be used in a model of thrombus formation, linking models defined on a variety of scales. On the finest scale we have the coagulation cascade (Figure 1, lower image), where a complex network of protein interactions contributes to thrombus formation through its promotion of fibrin formation and platelet responses. By utilising asymptotic techniques 
we can simplify the complexities of the coagulation cascade into a small number of equations or parameters, determining the response under different conditions that can then be incorporated into the next scale (i.e. Figure 1] upper left) 390 where platelets aggregate into a growing thrombus. 


\section{Coagulation in quiescent plasma with space distribution}

Having investigated the coagulation cascade utilising ordinary differential equations we now increase complexity by taking into account the diffusion of blood factors in plasma. The resulting reaction-diffusion system describe clot growth and emits travelling wave solutions. The concept of clot growth as a reaction-diffusion wave was introduced in the 1990s [85, 81, 66] starting an intensive investigation of wave dynamics and properties [82, 86, 87, 88, 89]. The main questions here are the determination of the conditions of wave initiation, the speed of its propagation and the mechanisms of its arrest. From a physiological point of view these determine the clot's size and differentiate normal, hemophilic and thrombotic plasma. In particular, low wave speed is specific for hemophilic patients while large wave speed can result in thrombosis.

In this section we present new methods for the investigation of the reactiondiffusion waves of blood coagulation allowing for the determination of the conditions of its initiation and its arrest, as well as the speed of clot propagation.

\subsection{Clot growth as a reaction-diffusion wave}

Self-sustained propagation of thrombin in plasma proceeds with constant speed and can be considered as traveling wave solutions of the reaction-diffusion system of equations describing the coagulation cascade:

$$
\frac{\partial u}{\partial t}=D \frac{\partial^{2} u}{\partial x^{2}}+F(u)
$$

where $u$ is the vector of concentrations of blood factors, $D$ is the diagonal matrix of diffusion coefficient, and $F(u)$ is the vector of reaction rates. Under some simplifying assumptions the full system can be reduced to the system of six equations for the concentrations $V_{5}, V_{8}, V_{9}, V_{10}, V_{11}$ and $T$ of active forms of the factors V, VIII, XI, IX and X, respectively, and thrombin 66, 85, 81. ${ }_{415}$ Then $u=\left(V_{5}, V_{8}, V_{9}, V_{10}, V_{11}, T\right)$. This system of equations should be completed by equations for fibrin production from fibrinogen (activated by thrombin) and 
fibrin polymer production. Since these equations do not influence thrombin production, system (2) can be considered independently.

Under the assumption of detailed equilibrium (large reaction constants) this ${ }_{420}$ system can be reduced to a single equation for the dimensionless thrombin concentration $\theta$ :

$$
\frac{\partial \theta}{\partial t}=d \frac{\partial^{2} \theta}{\partial x^{2}}+\alpha \theta^{3}(1-\theta)-\sigma \theta
$$

The second term in the right-hand side of this equation describes thrombin production and the last term its deactivation by antithrombin, $\sigma$ is proportional to the concentration of antithrombin.

425 Existence and stability of waves. It can be easily verified that the nonlinearity in equation (3) has three zeros if $\sigma$ is less than some critical value. Furthermore, the intermediate zero is unstable while the two others are stable. If $\sigma$ exceeds the critical value, then there is only one equilibrium $\sigma=0$, and the concentration of thrombin will necessarily vanish. Therefore, the bistable case with three zeros is biologically relevant. It is proved that the vector-function $F(u)$ in $(2)$ has the same number of zeros as the scalar equation, $u_{+}=0, u_{0}$, and $u_{-}$. The matrices $F^{\prime}\left(u_{ \pm}\right)$have all eigenvalues in the left-half plane, and the matrix $F^{\prime}\left(u_{0}\right)$ has a positive eigenvalue [90], 91]. In this case the thrombin free equilibrium $u_{+}$ is stable. Coagulation begins if initial thrombin concentration exceed some threshold level.

Let us note that the vector-function $F(u)$ satisfies the following condition:

$$
\frac{\partial F_{i}}{\partial u_{j}} \geq 0, \quad i, j=1, \ldots, 6, \quad i \neq j .
$$

Therefore, if we consider system (2) on the whole axis it has a travelling wave solution $u(x, t)=w(x-c t)$ 90, 91, 92, 93. Here $c$ is the wave speed, and the function $w(x)$ satisfies the equation

$$
D w^{\prime \prime}+c w^{\prime}+F(w)=0, \quad w( \pm \infty)=u_{ \pm} .
$$

This wave is unique up to translation in space and globally asymptotically stable in the following sense. Consider system (2) with initial conditions $u(x, 0)=$ 
$u_{0}(x)$ such that it is a monotonically decreasing (component-wise) function with the limits $u_{0}( \pm \infty)=u_{ \pm}$. Then the solution converges to the wave, $u(x, t) \rightarrow$ $w(x-c t)$ as $t \rightarrow \infty$.

Convergence to the wave may not hold if the initial condition $u_{0}(x)$ is not a monotone function. As is usually the case for bistable reaction-diffusion systems, it should exceed some threshold. System 2 has a stationary pulse solution, that is a positive stationary solution $w_{0}(x)$ with the zero limits at infinity [94, 95]. Moreover, such solution exists if and only if the wave speed is positive. If $u_{0}(x)>w_{0}(x)$ for all $x$, then the solution grows and converges to the wave. If $u_{0}(x)<w_{0}(x)$ for all $x$, then the solution decays and vanishes. Thus, the pulse solution determines the initial thrombin concentration that provides clot growth.

Thus, we obtain two conditions for clot growth. The first is the condition on the parameters which provide the positiveness of the wave speed. This condition is equivalent to the existence of the pulse solution. The second condition concerns the initial thrombin concentration. It should exceed the pulse solution. Hence there is a need to determine the wave speed and the conditions of wave initiation.

Speed of propagation. The wave speed $c$ for system (2) converges to the speed $c_{0}$ for the single equation (3) in the limit of large reaction rate constants [90, 91]. The speed $c_{0}$ for the single equation can be approximated by the narrow reaction zone method and by a piecewise linear approximation [91. They give a good approximation for the speed of the complete system and of the experimental results (Figure 3 ).

Initiation. Initiation of clot growth occurs at the injured vessel wall, due to the expression of tissue factor which forms a complex with factor VIIa (activated factor VII) subsequently activating factors IX and X, that are present in plasma. These factors promote the conversion of prothrombin into thrombin. Taking the initiation into account, instead of a simplified one-equation model (3) we obtain 

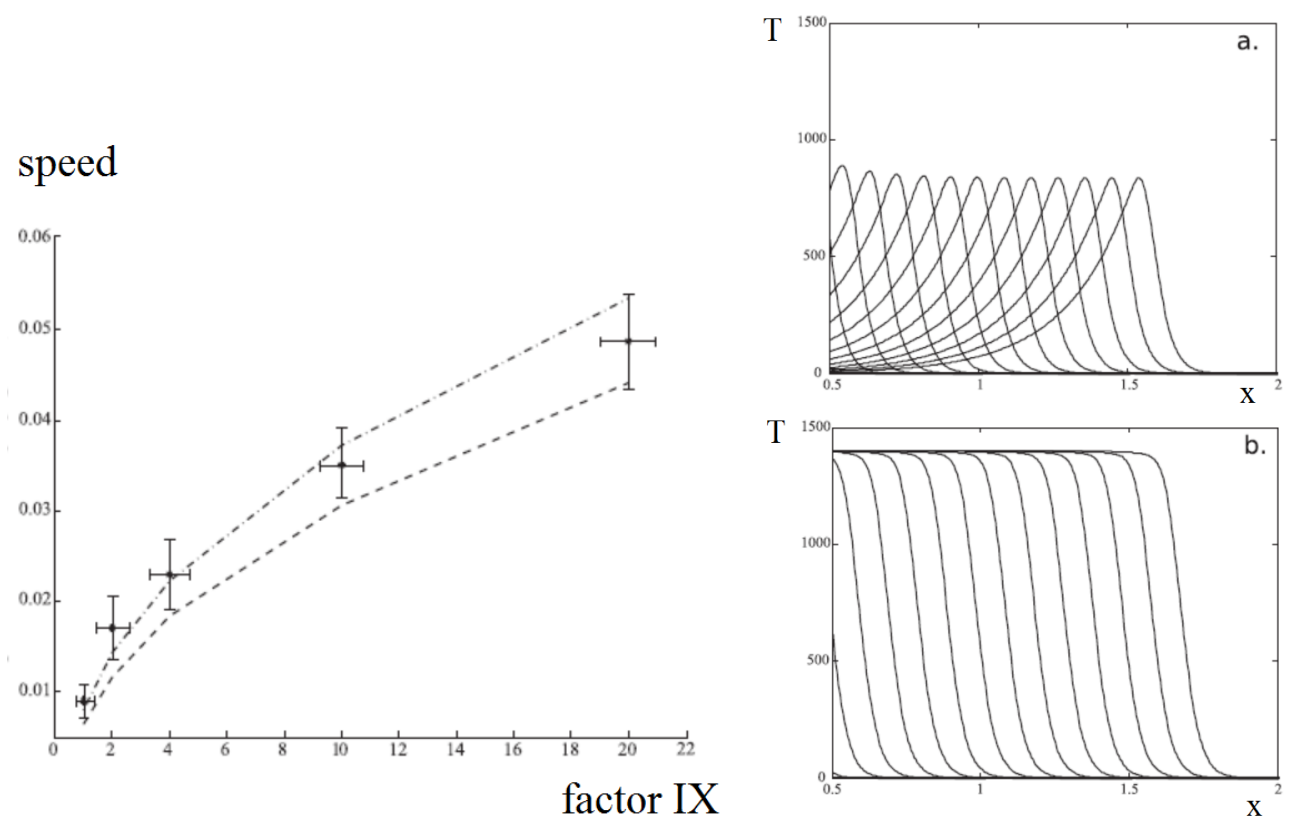

Figure 3: Left: speed of the thrombin wave propagation $(\mathrm{mm} / \mathrm{min})$ as a function of percentage of factor IX activity. Dots: experimental data [88]; dashed line: narrow reaction zone approximation; dash-dot line: piecewise linear approximation. Right: thrombin distribution at the consecutive moments of time for the complete model (a) and for the single equation 3 (b). Thrombin profile becomes monotone for the one-equation model because the equation for prothrombin is eliminated. Despite this difference, the speeds of propagation remain close. Reprinted with permission from [91].

the equation

$$
\frac{\partial \theta}{\partial t}=d \frac{\partial^{2} \theta}{\partial x^{2}}+\left(\beta e^{-r x}+\alpha \theta^{3}\right)(1-\theta)-\sigma \theta,
$$

where the term $\beta e^{-r x}$ describes the initiation by the wall located at $x=0$. If $\beta=0$, it is reduced to equation (3).

Similar to system (2), equation (3) considered on the whole axis has a pulse solution $\theta_{0}(x)$, that is a positive solution with zero limits at infinity. If the initial condition for equation (3) is greater than the pulse solution, then the solution of this equation converges to the wave, if the initial condition is less than $\theta_{0}(x)$, 
then the solution decays and converges to zero. The former corresponds to clot growth, while the latter to its failure. If the quantity of thrombin produced at the initiation stage $(\alpha=0)$ is greater than the threshold determined by the pulse solution $\theta_{0}(x)$ (for $x>0$ ), then the clot will grow. Otherwise, there will be some limited quantity of thrombin produced near the wall without further clot growth.

\section{2. $2 D$ growth and arrest}

Blood coagulation is inhibited via TFPI (tissue factor pathway inhibitor) present at the surface of endothelial cells, antithrombin, which is present in blood plasma, and protein $\mathrm{C}$, that is activated at the intact surface by a complex of thrombin and thrombomodulin and then diffuses in the vessel lumen. The concentration of antithrombin should be sufficiently low in order to allow the initiation and propagation of the thrombin wave. TFPI regulates the activation of the coagulation cascade at the vessel wall. Once propagation commences, neither of them (antithrombin, TFPI) cannot stop it. It can be verified that in the $1 \mathrm{D}$ model activated protein $\mathrm{C}$ does not stop the wave propagation neither.

Therefore, in order to describe clot growth arrest via activated protein $\mathrm{C}$ we need to consider 2D or 3D formulations, with an injured part of the wall where thrombin production is initiated and an intact part of the wall where protein $\mathrm{C}$ is activated. Protein $\mathrm{C}$ can then diffuse to the injured site and decelerate thrombin production. There is competition between self-sustained thrombin production and thrombin induced protein $\mathrm{C}$ activation, which downregulates thrombin production. For fixed values of other parameters, the result of this competition is determined by the injury size. If the injury size is sufficiently small, then the diffusion of activated protein $\mathrm{C}$ to the damaged site will be large enough to arrest thrombin production. If the injury is sufficiently large, then protein $\mathrm{C}$ does not stop wave propagation (Figure 4).

Thus, there are three regimes of clot growth in quiescent plasma: initiation without propagation, initiation with propagation and with growth arrest and initiation with propagation without growth arrest [96. The clot growth arrest 

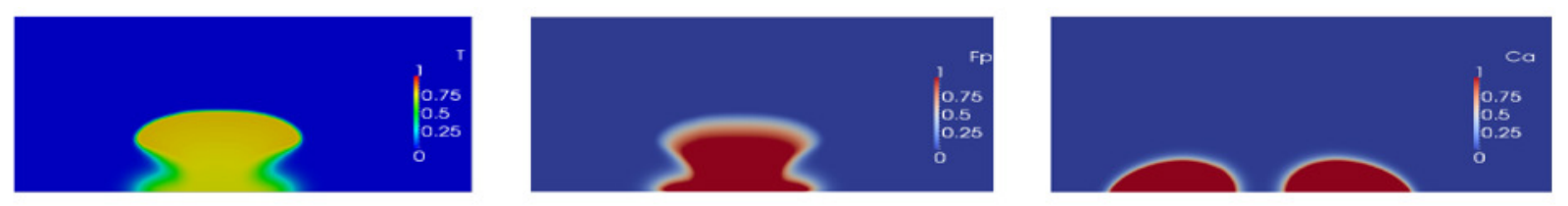

Figure 4: A snapshot of thrombin concentration, fibrin polymer and activated protein C. Thrombin production is initiated at the injury site (center of lower boundary) and diffuses to the intact wall to form a complex with thrombomodulin, activating protein $\mathrm{C}$. The latter diffuses back to the injury site and downregulates thrombin production. However, activated protein $\mathrm{C}$ is not sufficient here to fully arrest thrombin production and a thrombin wave escapes to the region where activated protein $\mathrm{C}$ does not reach.

critically depends on the lesion size with a sharp transition between complete 505 and partial occlusion [97. 


\section{Coagulation in flow without blood cells}

Increasing the complexity of the model we now introduce blood flow, excluding the influence of blood cells, which will be considered in the following sections. Blood flow brings inactive blood factors, such as prothrombin or fibclot. Conditions of clot initiation in flow and the distributions of blood factors during clot growth are studied in several works [98, 99, 100, 101, 102] and include comparisons with experiments undertaken in flow chambers [103, 104]. Coagulation with non-Newtonian flow is studied in [105, 106, 107].

515

In the previous section we discussed the mechanism of clot growth arrest by means of activated protein $\mathrm{C}$. We showed that if the damaged area of the blood vessel wall is sufficiently large then clot growth cannot be stopped, resulting in the complete vessel occlusion. Blood flow removes thrombin from the injured site and it represents an additional factor that can stop clot growth. Its influence is felt more dominant far from the vessel wall, where the flow velocity is larger and where the action of activated protein $\mathrm{C}$ is weaker.

A growing clot changes the geometry of blood flow and increases the complexity of the model. A possible approach to this problem is to consider it as 525 moves with time. In this case we need to trace this interface at every time step, solving Navier-Stokes equations in a complex domain with variable geometry.

The interaction of blood flow with clot can be taken into account through the addition of terms to the Navier-Stokes equations which describe flow deceleration inside fibrin mesh:

$$
\begin{gathered}
\rho\left(\frac{\partial \vec{v}}{\partial t}+\vec{v} \cdot \nabla \vec{v}\right)=-\nabla p+\mu \Delta \vec{v}-\mu K\left(F_{p}\right) \vec{v} \\
\nabla \vec{v}=0,
\end{gathered}
$$

where $K\left(F_{p}\right)=c F_{p}^{1.5}\left(1+56 F_{p}^{3}\right), F_{p}$ is the concentration of fibrin polymer and 

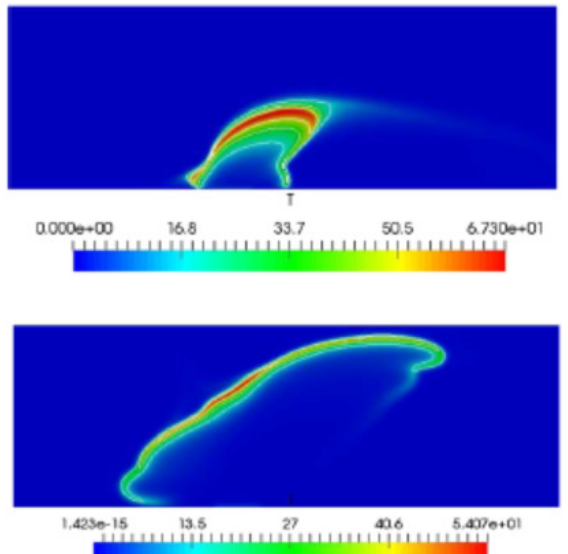
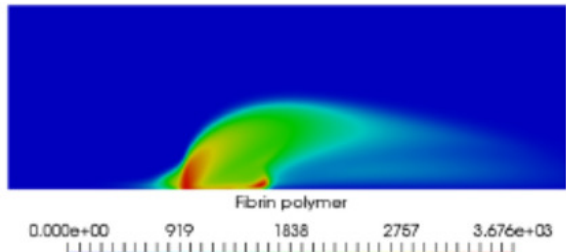

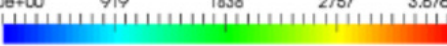

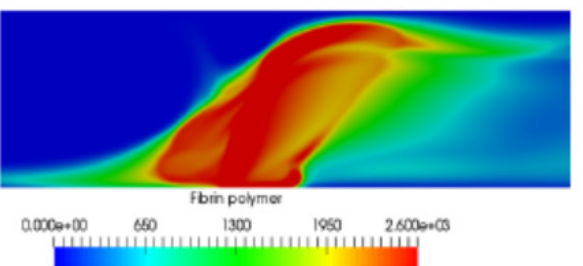

Figure 5: Snapshots of thrombin distribution (left) and fibrin distribution (right) in the beginning of clot growth (upper row) and before complete occlusion (lower row). Reprinted from [97. with permission.

$c$ is a numerical factor depending on the properties of fibrin mesh [108]. The advantage of this method is that the equations of fluid motion can be solved in the whole domain and do not need to determine the clot shape.

In order to describe the influence of blood flow on the growing clot, consider the rectangular domain $0 \leq x_{1} \leq L, 0 \leq x_{2} \leq H$ with no-slip boundary conditions at the lower and upper boundaries $x_{2}=0, H$. In the case of the given velocity field at the left and right boundaries $x_{1}=0, L$, it can be the parabolic flow: $v_{1}\left(x_{2}\right)=a x_{2}\left(H-x_{2}\right), v_{2}\left(x_{2}\right)=0$ with some given constant a. This boundary condition is convenient for small clots which do not strongly perturb blood flow. However in the case of large clots and, even more, in the case of complete occlusion, it will lead to unrealistic flow behavior. Indeed, if we take as an example the case where fluid cannot penetrate the clot (large $K\left(F_{p}\right)$ ), then mass conservation results in unbounded flow velocity as the clot approaches complete occlusion. To get a more realistic description of blood flow for large clots, instead of flow velocity the pressure difference is imposed. This means that the constant $a$ in the parabolic profile is not given, it is dependent on

time and is chosen in such a way that the difference between the mean pressure 
at $x=0$ and at $x=L$ is a given constant. In particular, $a$ can decrease in the process of clot growth, and can vanish in the case of complete occlusion and an impenetrable clot.
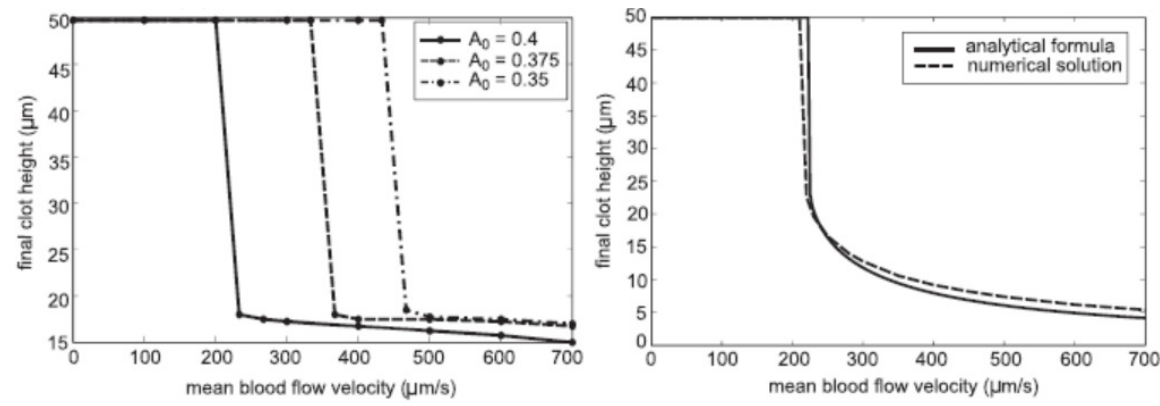

Figure 6: The final height of the clot in the case of different values of the initial blood flow velocity. Left: numerical simulations for different values of antithrombin concentration $A$ in plasma. Right: numerical (solid line) and analytical (dashed line) solutions of the simplified model for $A=0.4$. For both models, complete occlusion occurs for low flow velocities and partial occlusion for high velocities with a sharp transition between these two regimes. Reprinted from [109] with permission.

Equations for the concentrations of blood factors now contain convective terms. Examples of numerical simulations of clot growth in flow are shown in Figure 5 .

In order to obtain analytical estimates of clot growth, we consider the simplified equation for thrombin concentration

$$
\frac{\partial \theta}{\partial t}=D \frac{\partial^{2} \theta}{\partial x^{2}}+\Phi(\theta, x)
$$

where

$$
\Phi(\theta, x)=\alpha \theta^{3}(1-\theta)-\sigma(x) \theta,
$$

${ }_{555} \sigma(x)=\sigma_{0} A+\sigma_{1} v_{1}(x)$. This is similar to equation (3), with an additional term in $\sigma(x)$. The first term in the expression for $\sigma$ takes into account inhibition by antithrombin, the second term describes the loss of thrombin due to blood flow. Velocity profile $v_{1}(x)$ can be parabolic or it can take into account the 
influence of clot on flow velocity. For simplicity of notation we denote here by $x$ the variable $x_{2}$ perpendicular to the injured vessel wall.

Set $I(x)=\int_{0}^{\theta_{*}(x)} \Phi(\theta, x) d \theta$, where $\theta_{*}(x)$ is the largest zero of the function $\Phi(\theta, x)$ for fixed $x$. In the case without flow, where the function and the integral do not depend on $x$, condition $I>0$ provides the positiveness of the wave speed, that is the condition of clot growth. Since the thrombin wave front is sufficiently narrow, then a similar condition can be applied for every fixed $x$. Therefore, the thrombin wave propagates for the values of $x$ where $I(x)>0$. Condition $I(x)=0$ determines the maximal clot size if it less than $H$. Figure 6 shows the maximal clot size as a function of the maximal flow velocity obtained in direct numerical simulations of the 2D problem and in the approximate 1D equation 570 (9). The analytical condition through the sign of the integral and numerical solution of the simplified equation give a good approximation of the final clot size and of the conditions of complete occlusion.

A similar combination of numerical simulations and analytical estimates is used in [110] to study thrombosis treatment with warfarin. The influence of rheumatoid arthritis on thrombosis is investigated in [111.

Thus, in addition to three regimes of clot growth in quiescent plasma, there are two different possibilities depending on blood flow. In the case of propagation without arrest by activated protein $\mathrm{C}$, blood flow can stop clot growth or clot growth can continue leading to the complete occlusion. 


\section{Role of blood cells: Platelets}

Although models of plasma coagulation are important for understanding hemostatis mechanisms in veins and in vitro, the basis of arterial thrombosis is the attachment of blood platelets to the vessel wall injury [112, 113, 114. Platelets are the principal components of the hemostatic plug formed to arrest bleeding after an injury to the vascular wall. The most prominent of the hemostatic disorders is the thrombosis manifested by intravascular formation of platelet-fibrin clots that obstruct blood flow in the vessels. Attachment of platelets and their accumulation into thrombus is regulated by mechanical interactions with erythrocytes and vessel wall, by numerous chemical agents such as thrombin, or ADP, or prostaglandins, or collagen, as well as by an enormous network of intracellular signalling. The complexity of platelet aggregation and activation during thrombosis makes it an challenging goal for computational modelling: the problem involves complex hydrodynamics, cell adhesion mechanics, chemical cascades and a variety of different interplays between these 595 phenomena [115], some of which are yet to be understood.

\subsection{Platelet adhesion and aggregation in flow}

\subsubsection{Continuum-based models}

Blood platelets adhere to injured tissue to initiate the healing process in primary haemostasis. The accurate analysis of platelet-vessel wall interaction, tigations of the thrombus formation principles, formulating the prediction and observing the macroscopic morphological evolution of a thrombus over a long 
time-scale (up to several hours). The main problem here is the moving boundary condition at the thrombus surface, where both computational efforts (remeshing) and physical solutions (platelet adhesion, detachment rates, thrombus mechanics, etc.) should meet. Therefore, the construction of a mathematical model of the formation of a platelet aggregate with an adequate representation of its physical, chemical, and biological processes is an extremely complicated problem.

In works by Fogelson et al. 101, 116] cells were tracked via their concentrations $c_{i}$ the very same way as for the plasma coagulation factor. A system of reaction-diffusion-convection equations has the following form:

$$
\frac{\partial c_{i}}{\partial t}+(\mathbf{u} \cdot \nabla) c_{i}=D_{i} \Delta c_{i}+S_{i}\left(c_{i}\right)
$$

Here $D_{i}$ are the diffusion coefficients, which for platelets may depend on local hydrodynamic conditions (i.e. shear rate) [21, 117, and $S_{i}\left(c_{i}\right)$ are the reaction rates, representing here platelet activation, adhesion and aggregation. The moving boundary issue was resolved by domain decomposition approach, namely, the introduction of a "reaction zone", where all the cell-cell interactions, activation and binding take place, and advection zone (or bulk fluid), where no interactions occur, and only the passive streaming exists. The specially defined function of platelet inflow and outflow rates were used for fitting the concentrations on the boundary between zones. Advective and diffusive transport of platelets into or out of the reaction zone occurs at a rate proportional to the difference in the platelet concentration in the bulk fluid and in the reaction zone, and the value of a mass transfer coefficient. Platelets were considered to be in one of three states: i) unactivated and unattached (freely moving with the plasma), ii) activated and adherent to the subendotheleum, or iii) activated and bound to other platelets in the thrombus. However, such chemical agonists as ADP and TxA2 (normally released by platelets) were not accounted for explicitly in the model. Instead, the activation of platelets by contact with other activated platelets was introduced as an artificial rule. Besides, there is an experimental evidence that platelets can adhere to each other via GPIb-vWf 
bonds in unactivated state [118. The most strong assumption is that each chemical or platelet species were assumed to be well mixed (and homogeneous) in the reaction zone, which may not be the case in general. Whence this study is based upon approximations reasonable for small spatial domains and small thrombi, the models of large and occlusive thrombi (where inhomogeneities are important) will require a different approach.

In [119] the specific growth model defines the plug interface displacement based on the outcome of the biochemical model, that is, on the number of deposited bounded platelets on the injured part of the vessel wall. A system of reaction-diffusion-convection equations all coupled to each other was introduced to describe the evolution of concentration fields of all the species - proteins, solvable agonists and platelets, similar to [116]. However, the growing boundary of the thrombus was treated by Robin boundary condition that imposed a flux on the surface of thrombus/injury. It should be noted here that rules, upon which platelets attach to each other are different in [116] and [119, identifying the fact that the understanding of interactions and signalling between platelets in different states should be clarified by the means of detailed cell-based models and experimental work.

The most typical feature of early continuous thrombus models is the nonphysiological growth of a thrombus against the flow direction. To avoid this, deformations of the thrombus induced by the hydrodynamic drag should be taken into account. A special model describing the adhesion of platelets in terms of their concentration was constructed in terms of platelet concentration [120. It was assumed that the movement (rolling or sliding) of the already adherent platelets due the flow is one of the key processes in the thrombus formation. Specific terms accounting for thrombus deformation by the flow were introduced phenomenologically in order to obtain the experimentally observed shapes of thrombi in model (in 2D case):

$$
\frac{\partial C}{\partial t}=k_{\mathrm{adh}} \dot{\gamma}\left(1-\frac{C}{C_{\max }}\right)\left(\frac{C}{\lambda}+|\nabla C|\right)-k_{\mathrm{rol}} \nabla \cdot(v C)
$$

where $C_{\max }$ is the maximum value of the platelet concentration (dense packing), 
$\dot{\gamma}$ is the wall shear rate, $k_{\text {adh }}$ is the coefficient of platelet adhesion efficiency and ing thrombus. Depending on the size of injury, two distinct dynamic pathways of thrombosis were found: the formation of a nonocclusive plug, if injury length does not exceed the critical value, and the total occlusion of the vessel by the 
thrombus otherwise. Using only basic physical principles, not involving complex biochemical concepts, it was possibly to describe the switching mechanism between occlusive and non-occlusive thrombi in microvasculature.

As we see, the usage of continuum-based models in thrombosis and hemostasis has been fruitful, as it brought to life a number of ideas that encouraged experimental studies. Despite all the limitations, it would be considered as a handy instrument for both theoretical research and practice (engineering). However, some effects remained beyond the scope of continuum modelling of thrombosis nowadays. For instance, the correct constituitive model for thrombus mechanical and rheological properties is still missing. Also the proper choice of phenomenological constants is required. The internal structure of the thrombus that might be important for understanding the mechanisms of thrombosis regulation should be taken into account. Another issue that should be addressed in further studies is whether the platelet activation is an instant event or a multistage process, and how the platelet activation pathway influences the stability, density and final size of a thrombus.

\subsubsection{Effect of flow on platelet plug formation}

When the plug height is significant with respect to the vessel diameter, hydrodynamic forces play a crucial role in platelet thrombus growth. [119, 11. The interplay between platelet adhesion forces and the flow influences bounded platelets distribution, thrombus shape and the dynamics of the plug development. Let us consider here a simple model of platelet thrombus growth in a microvessel, taking into account both the erosion of the thrombus by the flow and the redistribution of the hydrodynamic velocities and pressure in circulatory system caused by the plug formation. Consider a hydraulic circuit presented in Fig.7.

The heart acts as a pump providing a systole-averaged constant flow rate $Q_{0}$. Let the thrombotic vessel to be presented as a series of resistive elements: a stenosed segment with resistance $R_{x}(t)$ and a healthy segment $R_{h}$. Let there be also a shunt consisting of a number of healthy vessels with overall resistance $R_{w}$. 


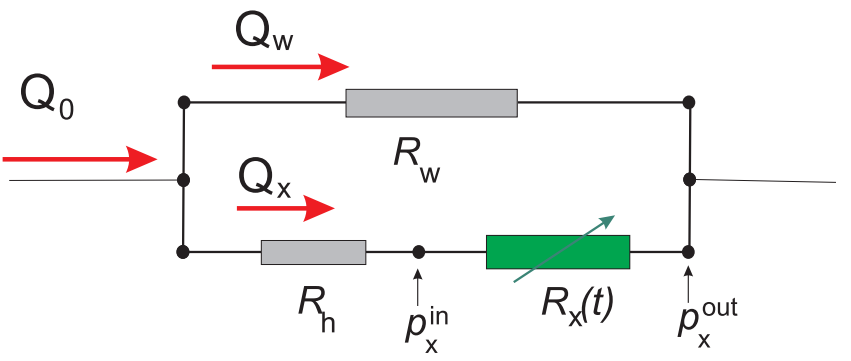

Figure 7: Hydraulic scheme of the thrombotic vessel (represented by "resistors" $R_{h}$ and $\left.R_{x}(t)\right)$ and the bypass $R_{w}$.

They provide bypass for blood flow as the thrombotic vessel is being occluded. Let us also approximate the thrombus by a ring characterized by its length $l=$ const, and inner radius $r(t)$. This is a rather strong assumption, but it would let us couple the hydrodynamics and thrombus growth and would allow the analytical consideration of the model. Therefore, one may simply write the expressions for pressure, blood flow rates and shear rates using Hagen-Poiseuille law $Q \cdot R=\Delta p$ and Kirchhoff's circuit laws. The increase of thrombus volume $d V$ in time $d t$ is caused by the inflow and adhesion of platelets through the surface of the plug/reaction zone $j_{\text {in }} A d t$, and the decrease of plug volume due to drag $-j_{\text {out }} A d t$ is governed by the erosion rate $j_{\text {out }} \propto \dot{\gamma}$ proportional to the shear hydrodynamic forces exerted on the surface of a plug. The equation for thrombus volume evolution reads

$$
\frac{d V}{d t} \approx\left(j_{\text {in }}-j_{\text {out }}\right) 2 \pi r l .
$$

Let for simplicity $j_{\text {in }}=$ const $=k_{0}$ and $j_{\text {out }}=\xi \dot{\gamma}$. Here $V=\pi\left(r_{0}^{2}-r^{2}\right) l$, so that $d V=-2 \pi r l d r$. By introducing dimensionless values, we obtain the equation for the lumen radius:

$$
-\frac{d r}{d t}=k_{0}\left(1-\frac{\dot{\gamma}}{\dot{\gamma}_{\mathrm{cr}}}\right)
$$

where wall shear rate $\dot{\gamma}$ is found by solving the Kirchhoff equations for the hydraulic circuit. Critical shear rate $\dot{\gamma}_{\mathrm{cr}}=k_{0} / \xi$ corresponds to zero growth rate of the plug. 
One can obtain a formal expression for the relation between the flow rate $Q_{x}$ and the applied pressure difference $\Delta p_{x}$ using Hagen-Poiseuille law and Kirchhoff circuit laws as follows:

$$
Q_{x}(t)=\frac{Q_{0} R_{w}}{R_{w}+R_{h}+R_{x}(t)}
$$

The pressure drop $\Delta p_{x}=p_{x}^{\text {in }}-p_{x}^{\text {out }}=Q_{x}(t) \cdot R_{x}(t)$ is found to be:

$$
\Delta p_{x}(t)=\frac{Q_{0} R_{w} R_{x}(t)}{R_{w}+R_{h}+R_{x}(t)} .
$$

For an axisymmetric thrombus (when thrombus growths over the whole perimeter of vessel's cross-section ) the lumen has a circular shape with inner radius $r(t)<r_{0}$, and the hydrodynamic problem may be solved exactly, so we find the hydraulic resistance of the thrombotic region

$$
R_{x}(t)=\frac{8 \eta l}{\pi r(t)^{4}}
$$

and the shear rate at surface of thrombus

$$
\dot{\gamma}=\frac{\Delta p_{x} \cdot r(t)}{2 \eta l},
$$

where $\eta$ is fluid viscosity. Since we do not consider RBCs in this example and this chapter, Newtonian fluid $\eta=$ const will be a good approximation.

For convenience we introduce the following dimensionless values: the relative thrombus length $\lambda=l / L$, scaled vessel's radius $\rho_{0}=r_{0} / L$, inner radius of the lumen $\rho=r / L$, and $\tau=t k_{0} / L$ is the dimensionless time. We define typical scale for hydraulic resistance as:

$$
[R]=\frac{8 \eta}{\pi L^{3}}
$$

As for the flow rate scale, it is convenient to express this value through combination of blood flow rate at the outlet of the heart $Q_{0}$ and the shunting hydraulic resistance $R_{w}:[Q]=Q_{0} R_{w} /[R]$. Pressure difference was thus scaled to a factor $[\Delta p]=Q_{0} R_{w}$. Shear rate in the stenosed vessel $\dot{\gamma}$ was scaled to the initial shear rate (when no thrombus yet grown)

$$
\dot{\gamma}_{0}=\frac{[\Delta p] \rho_{0}}{2 \eta}=\frac{Q_{0} R_{w} r_{0}}{2 \eta L},
$$


(a)

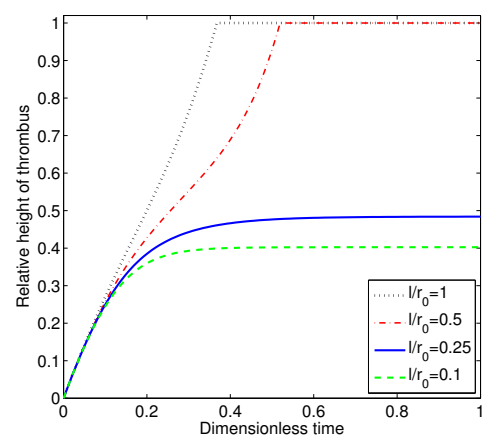

(b)

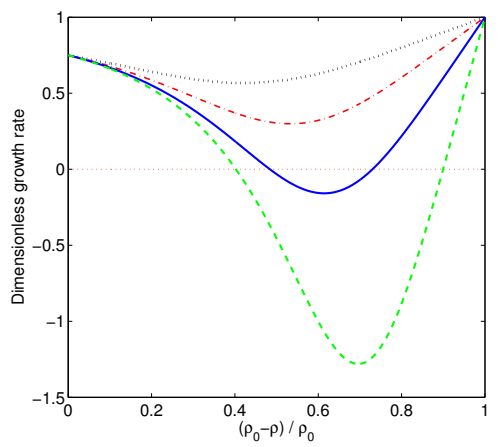

Figure 8: (a) Growth dynamics of axisymmetric thrombi for different thrombus lengths $\left(\rho_{0}=0.25, k_{0}=1.0, \xi=0.25, R_{w} /[R]=0.1\right)$. (b) Dimensionless growth rate $(-\dot{\rho})$ as a function of thrombus height for the same parameters.

so that dimensionless shear rate $G=\dot{\gamma} / \dot{\gamma}_{0}$. The equation 13 in dimensionless form reads

$$
-\dot{\rho}=1-G / G_{\mathrm{cr}}
$$

with $G_{\mathrm{cr}}=\dot{\gamma}_{\mathrm{cr}} / \dot{\gamma}_{0}$ and

$$
G=\frac{\dot{\gamma}}{\dot{\gamma}_{0}}=\frac{\left(\rho / \rho_{0}\right)}{\lambda+(1-\lambda)\left(\rho / \rho_{0}\right)^{4}+\rho^{4}\left(R_{w} /[R]\right)},
$$

The thrombus growth dynamics is presented in figure 8. We see that depending on the relative length of an injury $\lambda=l / L$, the thrombus may stop growing (if $\lambda$ is small enough) or keep growing until total occlusion of the vessel. The detailed analysis of the problem tells that the saddle-node bifurcation occurs in the system when $\lambda$ increases [11. There exists a critical thrombus length $\lambda_{\text {cr }}$ that differentiates the regimes of occlusion and thrombosis stoppage. For $\lambda>\lambda_{\text {cr }}$, the thrombus always blocks the vessel, while for $\lambda<\lambda_{\text {cr }}$ the thrombus reaches finite size. The value $\lambda_{\text {cr }}$ should be determined from the condition $G_{\max }=G_{\mathrm{cr}}$, where $G_{\max }$ is the peak value of the dimensionless shear rate. This value is found from the condition:

$$
\frac{d G}{d \rho}=0
$$


Thus we find the radius $\rho_{*}$, for which extremal shear rate $G_{\max }$ is achieved:

$$
\frac{\rho_{*}}{\rho_{0}}=\left[\frac{\lambda}{3(1-\lambda)+3 \rho_{0}^{4}\left(R_{w} /[R]\right)}\right]^{1 / 4}
$$

and

$$
\begin{gathered}
G_{\max }=\frac{3^{3 / 4}}{4 \lambda^{3 / 4}\left(1-\lambda+\rho_{0}^{4} R_{w} /[R]\right)^{1 / 4}}, \quad \text { if } \quad \lambda \leq \frac{3}{4}\left(1+\rho_{0}^{4} \frac{R_{w}}{[R]}\right), \\
G_{\max }=1, \quad \text { if } \quad \frac{3}{4}\left(1+\rho_{0}^{4} \frac{R_{w}}{[R]}\right)<\lambda<1 .
\end{gathered}
$$

Therefore, the formation of a stable finite thrombus in our model is possible for $\lambda<0.75\left(1+\rho_{0}^{4} R_{w} /[R]\right)$ (otherwise thrombus growth is not possible at all due to strong drag forces). And the condition $G_{\max }=G_{\mathrm{cr}}$ results in

$$
\lambda_{\mathrm{cr}}^{3}\left(1-\lambda_{\mathrm{cr}}+\rho_{0}^{4} \frac{R_{w}}{[R]}\right)=\frac{27}{256}\left(\frac{1}{G_{\mathrm{cr}}}\right)^{4} .
$$

Here $G_{\mathrm{cr}}=\left(k_{0} / \xi\right) / \dot{\gamma}_{0}$ is the combination of biological $\left(k_{0}, \xi\right)$ and hydrodynamic $\left(\dot{\gamma}_{0}\right)$ characteristics that defines the evolution of the system. Basically, $\left(k_{0} / \xi\right)$ is the ratio of platelet influx and drag-induced erosion. The solution of algebraic equation 26) determines critical thrombus length $\lambda_{\mathrm{cr}}=l_{\mathrm{cr}} / L$, so that if $\lambda>\lambda_{\mathrm{cr}}$, the thrombosis always proceeds to occlusion, and for $\lambda<\lambda_{\text {cr }}$ the thrombus reaches stable non-occlusive size. A saddle-node bifurcation occurs at $\lambda=\lambda_{\mathrm{cr}}$. Usually, $\rho_{0}^{4} R_{w} /[R] \ll 1$ in microvasculature, so that this term may be neglected, and the dynamics of the ring-shaped thrombus in our model is governed by $G_{\mathrm{cr}}$ parameter and thrombus length $\lambda$.

The results of a simple model suggest that the mechanism of thrombosis regulation in microvasculature rests on the balance between the platelet-aggregation rate $k_{0}$ and hydrodynamics-induced wash-off of the platelets from the plug. The principal conclusion is the existence of a threshold injury length demarcating regimes of occlusive thrombosis and growth stoppage. Strictly, the plasticity and deformability of a platelet aggregate escape from our scope in this consideration due to the model restrictions. Besides, length of the plug may change during growth. However, this elongation is usually moderate (smaller than an 
order of magnitude) and is not essential for microvascular thrombi. We also do not consider here a number of biological processes and features (platelet activation, fibrin growth, granule secretion, extent of damage, tissue-factor concentration, etc.) that take place inside the thrombus and basically cannot alter the surface-related hydrodynamic mechanisms considered here. These processes however may play an important role in biological regulation of thrombosis and hemostasis and should be addressed in further research: rheology and elasticity of a thrombus, vasoconstriction, platelet activation, platelet signaling inside the thrombus and plasma coagulation, etc.

Arterial walls are exposed to tangential shear stress and normal intraluminal pressure. Mechanical stimuli may cause a myogenic response: the variations in diameter of arteries or arterioles resulting from contraction and relaxation of smooth muscle cells in their walls [122, 123, 124]. This phenomenon, known as vasomotion, could be modulated by pressure changes [125, 126, and it is indeed the case during thrombosis and hemostasis. It is known that vasoconstriction of damaged arteries is one of the protective mechanisms, that takes place in the very first moments after the injury. It is particularly important in restraining acute haemorrhage in arteries: the constriction of the vessel lumen lessens the blood flow rate. In microvessels the vasomotion also affects the dynamics of thrombus growth in cases of a puncture-induced thrombosis (see for instance [114 and supplemental video 1 therein). The mathematical modelling of this phenomenon is a challenging task itself: both myogenic and metabolic autoregulation should be coupled to mechanical model of vessel walls and hemodynamics. A number of continuum-based models of vasoconstriction exist [127, 125, 123] and, hopefully, integration of this module into multiscale models of thrombosis and hemostasis will be possible in near future.

\subsubsection{Discrete models}

Whereas continuous models describe macroscale dynamics of platelet concentrations, it is reasonable to suggest that many aspects of thrombosis are better modelled as discrete events at the microscale. Modelling explicitly the 
transport, activation, and adhesion of platelets is crucial for predicting thrombus formation and growth following a thrombotic event in normal or pathological conditions. Particle-based discrete models are used in a variety of problems dealing with biological complexity, however computational costs may be tremendous in case of thrombosis as the number of cells in simulation may reach hundreds of thousands or greater depending on the dimensions of the problem.

Discrete methods may be subdivided in two classes: 1) coarse-grained approaches and 2) high-resolution cell models. The first class treats cells as particles interacting via a central potential and coupled to fluid usually via Stokes friction force [117, 10, 128, 129. It is possible to simulate the assembly of tens of thousands to millions of cells or particles with reasonable computational efforts. Although the details of cell shape, contact forces and adhesion are 775 simplified and sometimes neglected in coarse-grained approaches, one may still resolve the micro-structure of cell aggregates and emerging marcoscopic material properties. The second class of methods provides more details by the cost of computational complexity. Those models where platelets are represented as material points/spheres with a central potential are conceptually different from e.g. models accounting for each individual bond, cell shape and deformations, etc. 130, 131, 132, 133. In the latter case the spacial resolution that is required/accessible scales as $10 \mathrm{~nm}$, while for coarse-grained - as 1 micrometer. Consequently, computational efforts required for high-resolution models. We further discuss several examples of discrete models of platelet aggregation in flow starting with coarse-grained models.

A notable model was proposed by 134 with a stochastic cellular Potts model (CPM) used to describe cell-cell interactions. The CPM is a cell-level, energyminimization-based lattice model, which uses an effective energy (Hamiltonian) coupled with the local flow rate, to describe cell dynamics, adhesion, differentiation, division and hapoptosis. Given an effective energy one can calculate the resulting cell motion using Metropolis dynamics algorithm. Being the extension of idea of lattice automata, just like Lattice Boltzmann scheme used extensively nowadays to simulate viscous fluid flow in complex geometries [135, this scheme 
was developed for a number of biological problems including cell division, bacterial films [136], tissue growth, cell differentiation and others [137, 138]. The trial of CPM in thrombosis and hemostasis simulations was almost inevitable. In [134] it was assumed that activation of platelets occurs when the concentration of chemical components is higher than a specified threshold. The effective activator concentration was calculated as function of the three main agents (ADP, TxA2 and thrombin fIIa), accounting for the additive effect of the exceed of their concentration above certain thresholds:

$$
\Omega=\frac{[T x A 2]}{[T x A 2]_{\mathrm{th}}}+\frac{[A D P]}{[A D P]_{\mathrm{th}}}+\frac{[f I I a]}{[f I I a]_{\mathrm{th}}} .
$$

Despite biological complexity of this model, the shapes of thrombi were far from the experimentally observed ones. Therefore, reconsideration is required for numerous aspects in this field, including the rules for cell migration, thrombus deformation in response to the flow and retraction of platelet aggregate. Presumably, the separate case study is needed for platelet-platelet signaling, activation and procoagulant activity before incorporating these phenomena into a multiscale catch-all model.

Nowadays in a number of existing coarse-grained models of thrombosis the platelets are idealized as solid spheres or material particles with the same effective radius and/or hydrodynamic properties as those in the real life. Such an approach reduces the computational costs compared to full-cell shape-resolving simulation, while keeping the collective phenomena, crowding, jamming and granular media effects taken into account [139]. This granular nature of the thrombus or platelet aggregate usually escapes from the scope of continuum models. But, apparently, the mechanisms involved into thrombosis regulation and mechanical properties of thrombi depend strongly on the density and the inner structure of platelet aggregates.

One of the hypothetical mechanisms of the thrombus growth regulation is based on the interaction of the platelet- and plasma-dependent hemostasis. It was suggested that a fibrin-reinforced "core" is formed within the thrombus. This idea is supported by recent experimental data [114. This "core" is practi- 
cally non-destructible by the flow, however, the outer regions the platelet thrombus (above the fibrin core) are loosely packed and could be disrupted and removed by flow. This externalizes the inert fibrin that prevents further platelet attachment and stops further growth of a thrombus. This hypothesis is supported by a simulation study [10, 12] that employs a hybrid DPD-PDE model in order to grasp the interplay between platelet adhesion and plasma coagulation in microvascular flow conditions. Dissipative particle dynamics (DPD) was used to model plasma flow with platelets while fibrin concentration is described by a simplified reaction-diffusion-advection equation. Platelet activation and emergence of strong connections were modelled as a time-delayed strengthening of bonds, and platelets covered by fibrin with a sufficiently high concentration could not attach new platelets. The use of time dependent platelet adhesion force allowed the creation of a clot core, in which the forces between platelets are stronger than the ones between the newly connected platelets in the outer part of the clot. Similar approach to platelet adhesion was used by Mori et al. 129 with a distinction that the binding force between particles was modeled using the Voigt model (a spring with viscous damping). Different properties were introduced to consider the distinct behaviors of vWF-mediated and fibrinogenintegrin-mediated adhesion. Significant development of thrombus formation in height requires not only GPIb-vWf bonds, but also the activation of integrins. In general, despite its simplicity, this kind of models can give an insight into the mechanisms of clot growth and clot rupture.

Another example of hybrid particle-PDE multiscale models was proposed by Flamm et al. [128. It consists of 4 submodels: a lattice kinetic Monte Carlo tracking platelet motion and adhesion, a neural network model of platelet calcium signaling, a finite element method for calculation of concentration fields of ADP and TxA2 in plasma, and lattice Boltzmann submodel to determine the velocity field of the blood flow. Notable feature here is a neural network model trained on each donor's pairwise agonist scanning experiment and then embedded into a multiscale Monte Carlo simulation of donor-specific platelet deposition under flow. Such multiscale model could be feasible for patient- 
specific predictions of platelet deposition and drug response. It was the first such approach to make donor-specific predictions of platelet function under flow in the presence of both released ADP and thromboxane.

The initial adhesion of platelets on the thrombogenic area is governed by a variety of receptor-ligand interactions, such as a membrane receptor complex glycoprotein Ib-IX-V (or shortly GPIb) on platelets with von Willebrand Factor (vWF) multimeric protein. At low shear rates (below 1000/s), platelets adhere to the thrombogenic area through different pathways, relying on the exposed extracellular matrix proteins, while as shear rate increases, interactions between immobilized vWF and GPIb become exclusive in initializing platelet aggregation [112. The vWF proteins, which normally reside in a coiled state, tend to uncoil and expand in high-shear environments [140. This conformational change leads to enhanced adhesiveness of platelets. In recent work by Yazdani et al. [117] a shear-dependent platelet adhesive model has been proposed. The authors introduced an Eulerian-Lagrangian model where hemodynamics was solved on a fixed Eulerian grid (via Navier-Stokes equations), while platelets were tracked using a Lagrangian framework (via velocity-interpolation approach). The reactive transport of chemical species on a fixed Eulerian grid was also considered. The model of adhesion was based on the Morse potential with a shear-dependent potential well depth. The model is able to predict thrombus occlusion time with no significant computational cost using a data extracted from different experiments.

The role of platelet concentration is very important in coarse-grained simulations, especially when red blood cells are discarded from the model. The efficacy of platelet adhesion in shear flow is known to be substantially modulated by red blood cells 141, 20, 21. The near-wall excess (NWE) of platelets augments the frequency of platelet-wall collision, which aids in the hemostatic function of platelets [142, 143, 9]. A reasonable way to account for the platelet excess without RBCs is to set a higher homogeneous concentration of platelets in the inlet [16], or to prescribe the distribution of platelets according to experimental data 117. Besides that, a special boundary inflow and outflow conditions 
are required for platelet species in the model in order to maintain the constant

tion of particle shape and proximity of physical flow boundaries. The platelet's unique spheroidal shape provides heterogeneous, orientation-dependent rolling behavior which enhances cell-wall and cell-cell interactions, which are critical for initial stages of thrombus formation 144 . shape for its ability to adhere. But this work revealed the influence of thrombus porosity on cell dynamics near the thrombus. For thrombi with pores sufficiently large to contain a platelet, platelets did not pass over the thrombus, but 
instead penetrated into the thrombus where they became trapped. The median time that platelets spent in close proximity to the thrombus also was higher for more porous thrombi. These platelets would have a significantly increased time to respond to activation signals and firmly adhere to the thrombus via integrin-mediated bonds. The authors concluded that this may help to promote early growth of the thrombus. The importance of platelet-RBC collisions was stressed in this paper, indicating the fact that further simulation studies should account for this phenomenon.

Due to the computational costs of direct shape-resolving and bond-resolving simulations of platelet motion and adhesion, the bibliography is plenty of $2 \mathrm{D}$ models. Such models are able to simulate only small number of cells on a timescale of several seconds, but not the whole occlusive thrombus dynamics. Modern approaches use 3D hydrodynamic approaches and incorporate RBCs into consideration [146]. Limited applicability of platelet-only models discourages their development and application. However, these are promising for revealing the complexity of platelet-platelet adhesion mechanisms, their activation and signaling. And there is a lot of space for fundamental research in this subfield to the date.

\subsubsection{Modelling of ligand-receptor cell adhesion}

The adhesion and fragmentation of cells is ubiquitous in biological systems. The adhesive forces are composed of numerous physical processes including ligand- receptor binding kinetics, surface deformations, fluid flows, etc. Most of contemporary approaches rely on the idea that bond formation and breakage could be viewed as a statistical process modelled as a reversible chemical reaction between the populations of free membrane receptors (or cell adhesion molecules, CAMs) and their ligands. These reactions could be characterized by forward $k_{\text {on }}$ and reverse $k_{\text {off }}$ reaction rates. Most of the CAMs belong to four protein families: immunoglobulin superfamily, the integrins, the cadherins, and the selectins. There is distinction in their structure and mechanisms: usually the classifications distinguish between calcium-independent CAMs and calcium- 
dependent CAMs, some CAMs demonstrate high selectivity and require a specific ligand to attach (GPIb platelet receptor, ), others are less selective and may interact with many ligands (like GPIIb/IIIa of the platelets which can bind to vWf and fibrinogen). Nevertheless, the basic principles of their action could be described in a common way.

Bell was first to adapt the kinetic theory of the strength of solids and proposed a constitutive relation between dissociation rate and force [147]:

$$
k_{\mathrm{off}}=k_{\mathrm{off}}^{0} \exp \left(-F / F_{D}\right)
$$

assuming $k_{\mathrm{on}}$ constant. Here $F_{D}$ is the typical force required to apply for bond dissociation. This relation implies that the stronger the stretching of the bond, the shorter its mean lifetime. Another variation is to use a power law instead of the exponential law as proposed in [148, 149]. Also constitutive equations of the similar exponential force-dependent form have also been proposed for the binding affinity [150, 151]. Later Dembo and co-workers [152, 153] proposed a modification to original Bell's formula, including the ratio of elastic energy to $k T$ into exponent, instead of forces. The authors went further introducing the mathematical description for the so-called catch- and slip- bonds. It was done by assuming that a spring of the receptor has different stiffness in the bonded $(K)$ and non-bonded (transition) state $\left(K_{t s}\right)$ :

$$
\begin{gathered}
k_{\text {on }}=k_{\text {on }}^{0} \exp \left[-\frac{\beta K_{t s}\left(l-l_{0}\right)^{2}}{2}\right], \\
k_{\text {off }}=k_{\text {off }}^{0} \exp \left[\frac{\beta\left(K-K_{t s}\right)\left(l-l_{0}\right)^{2}}{2}\right],
\end{gathered}
$$

935 bond length. If $K_{t s}>K$, then the mean life-time $\left(1 / k_{\text {off }}\right)$ of the highly stretched bond will be longer than that for the unstressed bond $\left(1 / k_{\mathrm{off}}^{0}\right)$. This counterintuitive behaviour has been debated for many years until the existence of catchbonds was experimentally detected in bacteria 154 and a year later in leuko${ }_{940}$ cytes [155. It is believed that the physical principle of catch-bonds is related to conformational transitions in CAMs, similar to a Chinese finger trap. The 
exact mechanism is still being debated [156, 157, 158]. There are evidences that catch-bonds are also involved into initial platelet adhesion via GPIb-vWf bonds 159, 160.

Based on this findings, the numerical and semi-analytical model for leukocyte was proposed by Hammer and co-workers [161, 162, 163]. Within this approach the adhesive force and torque are calculated by integrating over the active bonds in the gap between adhering surfaces. The number of bonds per unit area $n_{b}$ at each particular moment of time could be found by solving the corresponding PDE:

$$
\frac{\partial n_{b}}{\partial t}+\mathbf{u} \cdot \nabla n_{b}=k_{\text {on }}\left(N_{l}-n_{b}\right)\left(N_{r}-n_{b}\right)-k_{\text {off }} n_{b}
$$

with the boundary conditions that bonds should vanish outside the adhesive contact zone. Here $N_{l}$ and $N_{r}$ are total numbers of ligands and receptors in the contact respectively. Within this continuum approach $n_{b}(\mathbf{x}, t)$ may be interpreted as the probability density of finding the active (attached) bond in a particular place at particular moment of time, since the process of bond formation and rupture is stochastic. In limiting case of weak affinity and abundant binding receptors on the surface of adhesion the bond density evolves as the following differential equation

$$
\frac{\partial n_{b}}{\partial t}+\mathbf{u} \cdot \nabla n_{b}=k_{\mathrm{on}} N_{l} N_{r}-k_{\mathrm{off}} n_{b}
$$

Let us consider as an example the problem of pulling apart (at constant velocity $U$ ) two parallel plates with ligand-receptor bonds forming in the gap. Introducing the dimensionless variables, the equation 32 reads:

$$
\frac{\partial N}{\partial T}=K_{\mathrm{on}}^{0} \cdot \exp \left[-K_{\mathrm{ts}}(H-1)^{2}\right]-K_{\mathrm{off}}^{0} \cdot \exp \left[\left(K-K_{\mathrm{ts}}\right)(H-1)^{2}\right] N .
$$

Here $N=n_{b} / N_{r}, K=\kappa l_{0}^{2} \beta /(2), K_{\mathrm{ts}}=\kappa_{\mathrm{ts}} l_{0}^{2} \beta /(2), K_{\mathrm{on}}^{0}=k_{\mathrm{on}}^{0} N_{l} l_{0} / U, K_{\mathrm{off}}^{0}=$ $k_{\text {off }}^{0} l_{0} / U, H=h / l_{0}$ and dimensionless time $T=t U / l_{0}$. The term $\mathbf{u} \cdot \nabla n_{b}$ vanishes due to geometry of the problem: the velocity is normal to plates and $n_{b}$ depends only on the lateral coordinates. We will solve this equation with respect to initial conditions $N(0)=0, H(0)=0$. The distance between plates evolves as $h(t)=U t$, or $H(T)=T$. In case of slow quasi-steady motion $U \ll l_{0} k_{\text {off }}^{0}$ the 
bond density may be approximated by the equilibrium value $N_{\text {eq }}$ obtained from the steady solution $(\partial N / \partial T=0)$ of $\mathrm{Eq} 33$.

$$
N_{\text {eq }}=K_{\text {eq }} \cdot \exp \left[-K(H-1)^{2}\right]
$$

where $K_{\mathrm{eq}}=K_{\mathrm{on}}^{0} / K_{\mathrm{off}}^{0}$ is the equilibrium constant for the binding reactions. This solution suggests that the number of bonds in the gap is mainly governed by the ratio of reaction rates and the distance between plates. Stiffer bonds (greater $K$ ) break at smaller distances. The transition state stiffness $K_{\mathrm{ts}}$ does not influence the equilibrium density of bonds, as the average time for a bond being in a free (transition) state is much smaller than typical time of the problem $l_{0} / U$. In general case, however, the solution will depend on $K_{\mathrm{ts}}$ leading to notable consequences for adhesion force. The general solution to 33 reads

$$
N(T)=\exp (-I(T)) \cdot\left(\int_{0}^{T} K_{\mathrm{on}}\left(T_{1}\right) e^{I\left(T_{1}\right)} d T_{1}\right)
$$

with

$$
\begin{gathered}
K_{\mathrm{on}}(\xi)=K_{\mathrm{on}}^{0} \cdot \exp \left[-K_{\mathrm{ts}}(\xi-1)^{2}\right], \\
K_{\mathrm{off}}(\xi)=K_{\mathrm{off}}^{0} \cdot \exp \left[\left(K-K_{\mathrm{ts}}\right)(\xi-1)^{2}\right], \\
I(T)=\int_{0}^{T} K_{\mathrm{off}}(\xi) d \xi .
\end{gathered}
$$

Results of direct numerical integration of Eq 33 are presented in Fig.9. different lines correspond to different transition state stiffness $K_{\mathrm{ts}}$. The slip bonds $K_{\text {ts }}<K$ eventually rupture as the plates are being separated leading to decrease of adhesion force density. In contrast, catch bonds $K_{\mathrm{ts}}>K$ remain longer in the stressed state than in the free state. Their lifetime increases as plates are being separated, this is why we see linear growth of adhesion force per unit area:

$$
\sigma_{z}=-n_{b}(t) \cdot \kappa\left[h(t)-l_{0}\right]
$$

or in dimensionless form

$$
F_{z}=\sigma_{z} l_{0} /\left(2 N_{r} k T\right)=-N(T) \cdot K[H(T)-1] .
$$



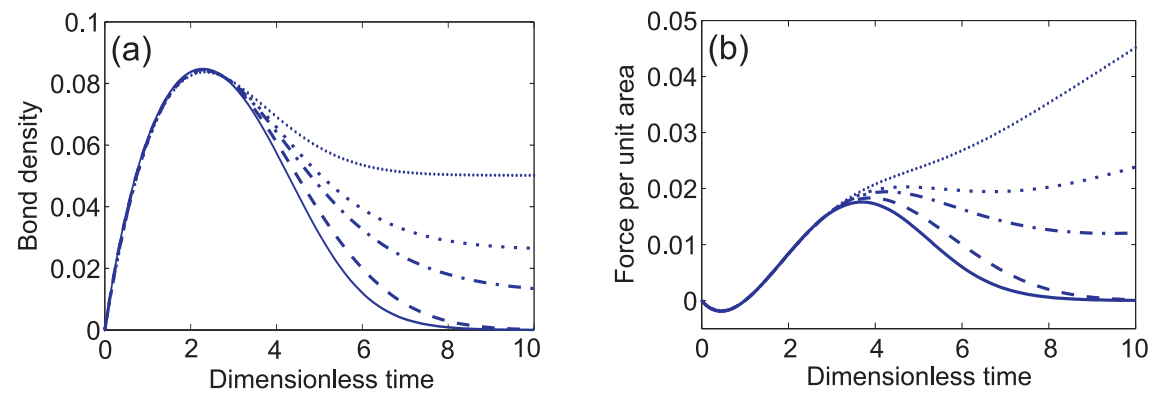

Figure 9: Numerical solution of the Eq33 (a) Density of bonds $N$ versus dimensionless time $T=t U / l_{0}$. (b) Resulting dimensionless normal adhesion force per unit area $\left(-\sigma_{z}\right) l_{0} \beta /\left(2 N_{r}\right)$ as a function of dimensionless time $T$. The parameters are as follows: $K_{\mathrm{on}}^{0}=0.1, K_{\mathrm{off}}^{0}=1.0$, $K=0.1$. Different lines correspond to different transient state stiffness parameter $K_{\mathrm{ts}}=0.05$ (solid), 0.09 (dashed), 0.13 (dash-dotted), 0.15 (small dots), 0.2 (bigger dots).

This simple phenomenological model grasps the unusually long-lasting bonds in leukocytes [161] and platelets [160]. However, this model is not the only possible one, and the mechanisms of catch-slip behavior may depend on the individual allosterical properties of a ligand-receptor pair. For instance, twopathway allosteric model [157, 164] relies on the assumption of the existence of two possible conformations, each of which decreases its lifetime with the force applied (as a normal slip bond does). But the transition between these states is biased so that the life-time of a lower-energy state becomes bigger with force. This model successfully explains the adhesion of Escherichia coli bacteria [157] via the FimH adhesin.

For the case of a shear (sliding) motion of plates, cylindrical and spherical particles a similar formalism could be allied. The results can be found in [165, 163, 166. In case of shear motion the bond tilting should be taken into account [165. By introducing a finite resistance to bond tilting (harmonic potential with stiffness $K_{\theta}$ ), the reaction rates in non-dimensional form read

$$
\begin{gathered}
K_{\mathrm{on}}=K_{\mathrm{on}}^{0} \cdot \exp \left[-K_{\mathrm{ts}}(L(H, \theta)-1)^{2}-K_{\theta} \theta^{2}\right], \\
K_{\mathrm{off}}=K_{\mathrm{off}}^{0} \cdot \exp \left[\left(K-K_{\mathrm{ts}}\right)(L(H, \theta)-1)^{2}\right]
\end{gathered}
$$


with $L(H, \theta)=H\left(1+\tan ^{2} \theta\right)^{1 / 2}, H=$ const and the bond density evolution equation with its boundary condition:

$$
\begin{gathered}
\sqrt{\epsilon} \cdot G \frac{\partial N}{\partial T}=-U \frac{\partial N}{\partial X}+K_{\mathrm{on}}-K_{\mathrm{off}} N, \\
X \rightarrow \infty, N \rightarrow 0,
\end{gathered}
$$

955

assuming that $U<0$ and there is no advection of bonds from downstream, $\epsilon=l_{0} / R$, where $R$ is the radius of a cell and $G$ is the dimensionless shear rate. Two distinct types of macroscale cell motion were predicted: cell rolling or cell sliding with bonds providing weak frictional resistance to sliding. The model predicts bistability between these two states.

Remarkably, such an approach is also applicable in Lagrangian simulation frameworks, like the immersed boundary method, the finite element method and the boundary integral method. Thus it could be coupled to hydrodynamics of cells of any complex geometry. Mody and King [131] analyzed the aggregation of blood platelets using the model inheriting from the pioneering works [152, 161]. Instead of solving the master equation for bond density, they accounted explicitly for every individual bond between Lagrangian surface points on platelet membranes. Only the adhesion via GPIb-vWf was considered. Since this type of bond relies on a solvable ligand vWf, a specific procedure was involved into the algorithm. Monte Carlo simulations were performed to test for the formation of new bonds between receptors on surface of one platelet, bound and free vWF ligand molecules, and receptors on another platelet. Bond formation and dissociation was tested using probability probability of bond formation $P_{\mathrm{on}}$ and the probability of bond breakage $P_{\text {off }}$ :

$$
\begin{gathered}
P_{\text {on }}=1-\exp \left(-k_{\text {on }} \Delta t\right), \\
P_{\text {off }}=1-\exp \left(-k_{\text {off }} \Delta t\right) .
\end{gathered}
$$

In 131] it was assumed that all A1 domains of vWF molecules were in active "bindable" state. However, the authors did not consider subpopulations of 
adhesive molecules that can switch to non-bindable state (GPIIb/IIIa), neither accounted for the catch-slip transitions in GPIb 160]. Insufficient quantifications of such phenomena in the literature preclude the development of models at this time. To date, mostly the Bell-like models have been used to describe the GPIb-vWf dissociation kinetics. Unfortunately, most of them do not account for the shear threshold effect exhibited by platelet interactions with surface-bound vWF [140], autoinhibition of vWf-A1 by the neighbouring vWf-A2 domain [167] and other microscopic phenomena that might be relevant for platelet aggregation during thrombosis. There is a critical need for the development of more detailed GPIb-vWf binding kinetic models with a greater physiological relevance. In addition, the models of activation and binding kinetics for platelet adhesion receptor GPIIb/IIIa are still to be implemented and validated.

\subsection{Coagulation in presence of platelets}

Vascular injury exposes the subendothelium and clot-promoting proteins: collagen, tissue factor and von Willebrand factor. They also activate the bound platelets causing them to release soluble chemical agonists into the plasma where these can activate other platelets and promote plasma clotting. Despite extensive research and great practical importance, there are only few models that attempt to simulate the interplay between plasma coagulation and platelet aggregation during thrombosis and hemostasis.

The influence of platelets on coagulation is not just chemical. The dense platelet aggregate in the site of injury provides a scaffold for the thrombin and the fibrin gel. The porosity of platelet aggregates slows down the flow velocities 985 and diminished the wash-out of plasma coagulation factors - thus in arterial conditions with high flow velocities the effective coagulation is impossible without platelets. The studies by Fogelson et al. indicate that at least for small injuries the flow-mediated dilution is the most potent inhibitor of the plasma clotting cascade [101, 116]. It was found that the importance of the thrombin-fXIa-fIXa feedback loop to robust thrombin production depends on the concentration of platelets in the blood near the injury [116]. In their model the authors assumed 
that each chemical or platelet species were well mixed in the reaction zone. The concentration of each protein satisfies an ordinary differential equation (ODE) that includes terms for reactions; the same approach was used for platelets. It was assumed that the reactions could occur in a reaction zone above a small patch of exposed subendothelium, and the height of the reaction zone was able to increase together with the total volume of platelets in the thrombus. Advection of species has been accounted only away from the reaction zone, which may be reasonable for the conditions inside stable platelet aggregates, where flow is hindered, or under venous conditions. These approximations reasonable for small thrombi are not applicable to larger thrombi that grow substantially into the vessel lumen. For larger injuries, in order to account accurately for the interaction between growing thrombus and the flow, a more complex spatialtemporal model is required. Interestingly, in the model by Fogelson et al. the membranes of activated platelets provided coagulation reactions for factors and their complexes (e.g. dimerisation of fXI or fXIa).
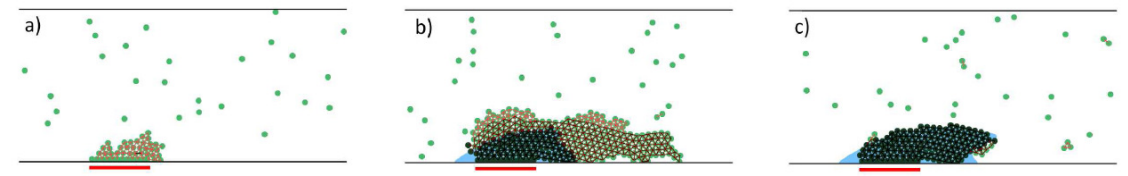

Figure 10: Numerical simulations of clot growth with the DPD-PDE method. Blood flow and platelets (green dots) are modeled with a particle (DPD) methods, the distribution of blood factors with reaction-diffusion-advection equations. Clot growth begins with platelet aggregation. They protect the injury site from the flow allowing the coagulation cascade to start (a). Fibrin network (blue) forms inside the platelet aggregate and reinforces the clot (b). The outer part of the platelet aggregate is removed by the flow giving its final size and shape (c). Reprinted with permission from [12].

In a hybrid DPD-PDE model by Tossenberger et al. [10, 12] the fibrin gel formation was modelled along with particle dynamic simulation of plasma flow and platelet adhesion. Because of the complexity of the coagulation process, the authors implemented a simplified model of the coagulation cascade where 
the distribution of blood factors are described by reaction-diffusion-advection equations. Several techniques, such as an introduction of the exchange period between DPD and PDE solvers, bilinear interpolation for concentration fields, etc., were introduced to couple the mechanical and chemical blocks of the model.

The model shows the following clot growth process. At first, an early platelet aggregate forms at the injury site, which is necessary to protect the concentrations of blood factors (especially thrombin) not to be taken by the flow (Figure 10, a). Let us note that the first platelet aggregate can form before platelet activation. Their activation leading to the reinforcement of their connections occurs already in the clot. In that way blood coagulation can occur inside of the platelet clot. As a final product of blood coagulation, fibrin polymer is produced, reinforcing the inner part of the clot, thus forming the clot core (Figure 10. b). The clot core, which is more stable, can support further clot growth. As the clot grows, the blood vessel becomes narrower and the flow shear rate increases at the top of the clot. Due to the increased shear stress the softer outer parts of the clot, which are not covered by fibrin polymer, are one by one detached and taken by the flow. This process continues until clot stops growing (Figure 10, c). The hybrid model in this work is simple from biological point of view, but as such offers some useful insights in flow-thrombus-protein interaction and serves as a good base for future model development.

The most blurry issues in the field of platelet-plasma interactions during thrombosis are related to platelet activation and signal transduction. Activation of each platelet that occurs at the earliest stages of the thrombus formation is an extremely complicated process that includes shape change, granule secretion, etc., and is regulated by an immense signaling network that is only partially deciphered. This module does not require hydrodynamics but is extremely challenging from the biochemical point of view: most reactions are poorly known, and their parameters have not been determined. As for the network of blood coagulation, this part is well-studied, except the poorly known mechanisms of the membrane-dependent reactions [8] and in-flow fibrin polymerization dynamics 98. 


\section{Role of blood cells: Erythrocytes}

\subsection{Continuum models}

High concentration of red blood cells (RBCs) gives rise to the complex nonNewtonian rheology of blood, as well as to collective effects from cell-cell collisions.

On the one hand, PDE-based classical hydrodynamic-concentration models could be used by modifying the Navier-Stokes equation with phenomenological non-Newtonian viscous stress-tensor [168, 169], or a constitutive equation [170]. The model will remain relatively simple, efficient and scalable. The rheology, however, is not the main complexity that RBCs bring to the problem of thrombosis, and in most thrombosis-related cases non-Newtonian properties of blood may be neglected [169. Which is more important, it has been experimentally discovered [141] that platelets accumulate on the adhesive substrate faster in presence of erythrocytes, than in platelet reach plasma or buffer, due to platelet redistribution in blood vessel caused by their mechanical interaction with RBCs [9. In 11] the implicit effect of RBCs has been accounted by the analytically approximated phenomenological dependence [20, 21] of platelet accumulation rate on wall shear rate. This non-linear effect of RBCs on platelet adhesion appeared to be important for hydrodynamic regulation of thrombus growth in microvasculature.

Only few works use continuum-based approach with implicit accounting of RBC effect on thrombosis [119, 171, 20]. Such models present spatial and temporal evolution of a thrombus via concentration functions (for both cells and biochemical agents), while effects of RBCs are accounted via effective diffusivities [171, 20] or by adjusting inlet platelet concentration profile so that platelets accumulate near vessel wall [119]. Due to computational efficacy, these approaches are able to track the dynamics of thrombosis in larger vessels on a timescale of several minutes, even hours. Yet the important constants and parameters (e.g. diffusivity of platelets, thrombus elastic moduli and porosity, platelet accumulation rates, etc.) could mostly be obtained by fitting experimental data. Thus, 
we see that the internal physics and biomechanics of thrombosis and hemostasis may escape from the scope due to phenomenological assumptions. Cell distribution across blood vessels, platelet activation and signalling, crack formation in platelet aggregates, and RBC collisions with thrombus might be important in understanding mechanisms of thrombosis regulation. The physical backgrounds of some effects remain unclear, until detailed cell-cell interactions are considered explicitly. Although continuum models fail to provide mechanistic picture of RBC impact on thrombosis, nevertheless they could be tuned to reproduce the correct phenomenology. The main advantage is their scalability (both in time and space) and easy implementation on the basis of CFD numerical methods. Due to its relative simplicity, this class of methods is the first candidate to engineering and medical applications.

On the other hand, modelling blood as a suspension of RBCs allows one to inherently capture important rheological and transport properties of blood ab initio. Such direct simulation approach is considered to be appropriate in a number of studies, where the number of interacting objects (e.g. colliding blood cells) is large, but continuum approach is not sufficient. Blood rheology is probably the most notable and famous example [172, 173, since it depends on cell-cell and cell-fluid interaction and could only be reproduced correctly if blood cells and their mutual collisions are modelled explicitly. Another example important for thrombosis and hemostasis is the phenomenon of blood platelet margination to the regions nearby the vessel wall $9,174,175,176$. This effect is interconnected with important biomechanical phenomena like the Fahraeus and Fahraeus-Lindqvist effects (caused by formation of RBC-depleted layer near wall). The effect of hematocrit on platelet initial adhesion has been numerically characterized [146] using 3D cell-resolving simulation.

Each of these effects may have an impact on thrombus growth: Non-Newtonian rheology is important in low-shear conditions [177, 169] of venous thrombosis, while the formation of RBC-depleted layer, platelet margination and plateletRBC collisions [20, 21] are crucial for arterial and microvascular thrombosis. Complexity of the model and of its numerical realization should be appropriate 
to the questions under investigation. Depending on the resolution of a simulation, on how detailed RBCs, their shape and mechanics are presented in the model, one could distinguish between coarse-grained and full-cell approaches.

\subsection{Coarse-grained models}

To this class of intermediate approaches we attribute any particle-based simulation, which does not describe the exact mechanics and shapes of erythrocytes and platelets. Models of this kind occupy an intermediate niche between continuum (macroscale) and cell-based (microscale) levels.

Numerous methods have been proposed to model whole-blood flows with conventional coarse-grained methods, such as Dissipative Particle Dynamics (DPD) [178 and Smoothed-Particle Hydrodynamics (SPH) [179] and others. Whence nowadays it is obvious that pure particle dynamics (that means that plasma is also presented as a particle ensemble) for whole-blood simulations is tremendously costly from computational point of view, modern coarse-grained approaches are hybrid: they combine continuum description for plasma with particulate description for cells [180, 181, 182].

It has been seen that various mechanical factors are involved in the regulation of primary thrombus formation through a series of physiological and biochemical processes, including blood flow and intercellular molecular bonds. Nowadays, it has become clear that RBCs influence thrombus formation mechanically (apart from rheology) in at least two ways: 1) enhancing platelet adhesion by pushing them to the wall of the vessel, 2) colliding into growing thrombus.

The latter one has been addressed via coarse-grained modelling [182, where RBCs had been represented as elastic spheres either soft of rigid. The results show that when red blood cells constrain the vertical growth of platelet thrombus and the platelet aggregate spread more horizontally compared to zero hematocrit case. This work pointed out the necessity of considering the presence of red blood cells when investigating the mechanism of thrombus formation, and also supported the idea that growth of a thrombus in vertical direction may be hindered by its mechanical interaction with streaming blood [11. 
The impact of RBC-platelet collisions on platelet margination to vessel walls has also been addressed via coarse-grained approaches. In [181] authors take erythrocytes into account implicitly, guided by experimental observations, as an effective "diffusion". They added a random step (increment) to each platelet's motion during each iteration. The alternative implicit way to incorporate the effect of the red blood cells also has been proposed in [181]: to exert a random force on each platelet, pushing it to the wall. However, choosing random force vectors and distributing the forces over the platelet's surface appeared to be not a very obvious procedure.

Another example [180] uses spherical impenetrable RBC-like and plateletlike colloids to reproduce whole blood. Using hydrodynamic simulations of such a simple model, authors showed that the adhesion probability of platelets to von Willebrand factor is strongly and nonlinearly dependent on hematocrit and flow rate, and that the origin of such behaviour is in the distribution of platelets. In other words, it appeared that the understanding of blood cell redistribution mechanisms does not require the exact mechanics of RBCs and platelets. This achievement is quite encouraging for coarse-grained modelling.

Overall, the coarse-grained modelling of thrombosis and hemostasis is limited nowadays. This class of methods, being a profitable trade-off between full-continuum and full-cell models, is able to cover partly the disadvantageous phenomenology of continuum models, yet raises computational costs. Since these models could not reproduce correct blood rheology (due to simplifications of RBCs shape and elasticity) they do not find applications in engineering and are being underestimated. However, these approaches have proven already the capability to reveal and demonstrate basic mechanisms of RBC influence on thrombosis. Therefore, coarse-grained models might be considered as a complementary illustrative tool in addition to the real-life experiments.

\subsection{Full-cell models}

The hypothesis of the formation of RBC-depleted layer near wall was recently supported by the computer simulations [9], see Fig, 11. The mechanism of this 
effect is believed to be caused by the interplay between the deformability of RBCs and the hydrodynamic Segre-Silberberg effect [183, 184]. As shown in Fig 12, the repulsion of RBCs from vessel walls is likely to increase platelet concentration in the near-wall region, where their physiological function should be fulfilled. This mechanism could only be numerically studied by "full-cell" models that resolve the actual shapes and reproduce the realistic elasticity of the RBCs and platelets. Being advantageous for the investigation of cell-cell and cell-wall collision dynamics, these ab initio simulations are very resourcedemanding. Let us now discuss the advances, challenges and some technical details of such full-cell hemodynamic models.
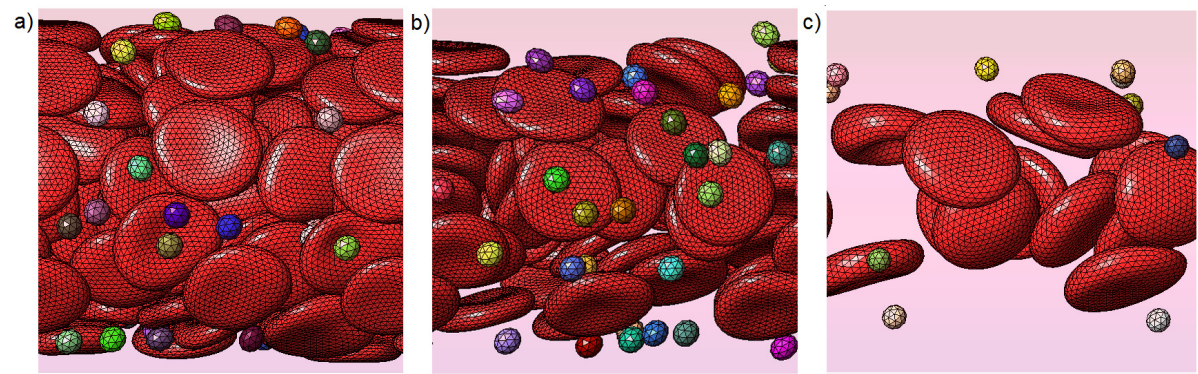

Figure 11: Snapshots of numerical simulations showing erythrocytes and platelets in the flow in cylindrical channel for three different values of $\mathrm{Ht}$ : a) $40 \%$, b) $20 \%$, c) $10 \%$. Blood plasma particles, which occupy the remaining volume of the channel, are not shown. Reprinted with permission from $[9]$.

Nowadays the primary tools for micro-scale cell shape-resolving hemodynamic simulations are as follows: the Immersed boundary method (IB), BoundaryIntegral method (BIM) and variations of mesoscopic Particle Dynamics. Mostly these models comprise of three principal blocks: viscous fluid (blood plasma), cell or cell membranes and coupling scheme that establishes mechanical connection between cells and fluid and assures the momentum conservation.

A number of works use different combinations of these techniques to describe motion of objects immersed in viscous incompressible fluid. For instance, blood rheology has been addressed [177, indicating that coin-like RBC aggre- 

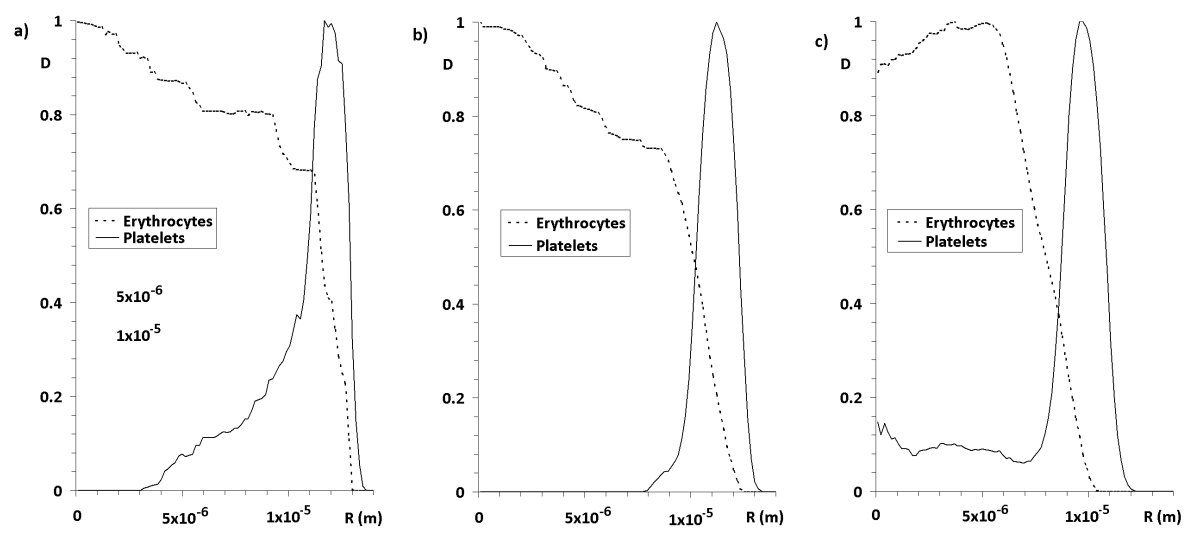

Figure 12: Erythrocyte (solid line) and platelet (dashed line) distributions as a function of distance from the axis for three values of $\mathrm{Ht}$ : a) $40 \%$, b) $20 \%$, c) $10 \%$. Reprinted with permission from 9 .

gation is responsible for increased apparent blood viscosity at small shear rates. Platelet margination to vessel walls has also been studied. Thus, [185] addressed the importance of platelet shape via front-tracking/immersed boundary method. With Boundary Integral simulations [146] supported the hypothesis that RBC-platelet collision being involved into platelet adhesion and thrombus formation. In 186, 145 on using 2-dimensional Lattice-Boltzmann-ImmersedBoundary method it was demonstrated that RBCs are responsible for the development of platelet near-wall excess and the mechanism for platelet margination is based on excluded volume effects. Using similar approach, [176] discovered that platelet clustering in aneurysms depends on aneurysm's geometry. Most efficient are models with hybrid approaches where fluid is treated as a continuum and cell membranes are presented as a mesh of particles.

\subsubsection{Spectrin mesh model of RBCs}

Every model that deals with erythrocytes at this level of details includes the well-validated membrane constitutive model. Most of contemporary hemodynamic simulations follow the pioneering works by Skalak et al. 187, 188, 189, 190, in which the mechanics of RBC's membrane was characterized and a 
model of spectrin network for RBC's skeleton was suggested [189, 190]. Later a coarse-graining procedure was developed [191] allowing for an RBC to be described with arbitrary number of vertices. The 3D spectrin-mesh model for RBC was proposed [192, 193, 194, 195, 191], which by this moment has become a well-validated standard in hemodynamics. The model accounts for stretching elasticity, bending rigidity, conservation of volume and surface. It is usually formulated in terms of Helmholtz free energy of the membrane:

$$
\begin{gathered}
W\left(\mathbf{x}_{n}\right)=W_{\mathrm{WLC}}+W_{\text {bending }}+W_{\text {volume }}+W_{\text {area }}, \\
W_{\mathrm{WLC}}=\sum_{\text {edges }} k_{\mathrm{WLC}} \frac{3 \xi_{i}^{2}-2 \xi_{i}^{3}}{1-\xi_{i}}+\sum_{\text {edges }} \frac{k_{\text {rep }}}{L_{i}}, \\
W_{\text {bending }}=\sum_{\text {adjacentfaces }} k_{b} \cdot\left[1-\cos \left(\theta_{a b}-\theta_{0}\right)\right], \\
W_{\text {volume }}=k_{v} \frac{\left(\Omega-\Omega_{0}\right)^{2}}{\Omega_{0}}, \\
W_{\text {area }}=k_{\text {ag }} \frac{\left(S-S_{0}\right)^{2}}{S_{0}}+\sum_{\text {faces }} k_{\text {al }} \frac{\left(A_{k}-A_{k 0}\right)^{2}}{A_{k 0}} .
\end{gathered}
$$

Here $\xi_{i}=L_{i} / L_{\max }$ is the relative deformation of an $i$-th edge, $A_{k}$ is the area of a $k$-th face, $\theta_{a b}$ is the angle between two adjacent faces $a$ and $b$. The force acting on $i$-th vertex is derived as $\mathbf{F}_{i}=-\partial W\left(\mathbf{x}_{n}\right) / \partial \mathbf{x}_{n}$.

A set of optical-tweezers experiments on RBCs by Suresh et al. [193, 194, [196, 197] is often used to choose the parameters of membranes (bending and stretching elastic moduli). The usual validation runs to assure that the RBC mechanics is correctly formulated in a model include [198: 1) the simulation of optical-tweezers stretching with comparison to experiments; 2) the shear test implying that RBC must change its tumbling motion for tank-threading at around 1000 /s. The biconcave shape of RBCs has also been digitized and used for initial meshing of cells in a model [198].

Worthy to notice that the viscosity of RBC interior is usually assumed to be equal to the one of the blood plasma. This is not strictly correct, but the 


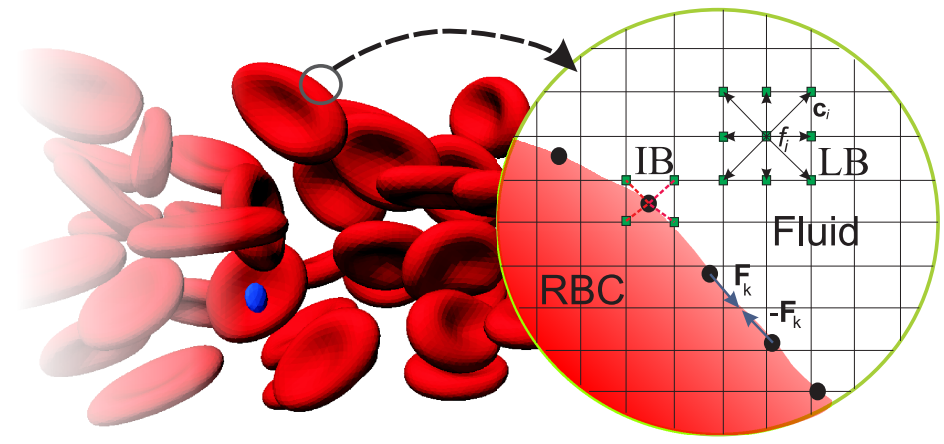

Figure 13: A scheme of the Lattice Boltzmann- Immersed Boundary simulation method for blood flows.

assumption reduces the model's complexity drastically: single-phase viscous fluid dynamics is easier to compute compared to two-phase fluid dynamics. Only a limited number of works account for a viscosity contrast in RBCs in their models [199]. In particular, it was shown that the transition from tank-treading to tumbling motion can be triggered by the ratio between internal and external fluid viscosities [199], influencing the rheology of the modelled suspension.

\subsubsection{Technical details: LBM-IB approach}

One of the possible schemes is based on the combination of immersed boundary and lattice Boltzmann methods (IB-LBM) 186, 200, 145, 201, 135, 202, 198, 203. Within such a framework RBCs are represented as a mesh of Langrangian surface points, interacting via a constitutive elastic membrane model (Fig. 13). LBM is employed to calculate velocities in the fluid phase. Its main advantages are that it converges if Reynolds and Mach numbers are smaller that unity, regardless of a node-mesh structure (thus, no need to refine fluid nodes mesh in complex geometry, etc.) and a relatively simple parallelism. LBM is coupled to the suspended RBCs with the immersed boundary method via the procedure of interpolation of velocities and forces between movable membrane nodes and stationary lattice nodes.

Basically, LBM is used as a fast solver for hydrodynamic equations, that inherits from lattice gas automata simulations. The method rests upon the 
kinetic equation that describes the spacial-temporal changes of a one-particle distribution function [135]. In other words, the main quantity $f(\mathbf{x}, \mathbf{c}, t)$ corresponds to the probability distribution of finding fluid particles (molecules) in site $\mathbf{x}$, at time $t$, moving with velocity $\mathbf{c}$. Being discretised in a 6 -dimensional phase space via a regular mesh of spacial sites $\{\mathbf{x}\}$ and lattice velocities $\left\{\mathbf{c}_{i}\right\}$, this function $f_{i}(\mathbf{x}, t)$ obeys the following equation:

$$
f_{i}\left(\mathbf{x}+\mathbf{c}_{i} \Delta t, t+\Delta t\right)=f_{i}(\mathbf{x}, t)+\Omega_{i}(\mathbf{x}, t),
$$

and the evolution of the system could be found by consequent iterations. Here $f_{i}(\mathbf{x}, t) \equiv f_{i}\left(\mathbf{x}, \mathbf{c}_{i}, t\right)$. Different schemes and lattices were proposed. The most widespread for 3D simulations is D3Q19 scheme, which means that 3-dimensional square lattice of spacial (Eulerian) sites are used with 19 unity velocity vectors $\mathbf{c}_{i}$. The most intriguing part is the collision function, that determines the interaction of fluid particles with each other, thus governing the macroscopic properties of the media. For viscous incompressible fluid the single-relaxation time approximation (or Bhatnagar-Gross-Krook approximation) is usually preferred:

$$
\Omega_{i}(\mathbf{x}, t)=-\frac{1}{\tau}\left(f_{i}(\mathbf{x}, t)-f_{i}^{\mathrm{eq}}(\mathbf{x}, t)\right)+\tilde{\Omega}_{i}(\mathbf{x}, t) .
$$

Here the relaxation time $\tau$ unequivocally defines the kinetic viscosity of the fluid $\nu=(\tau-0.5) c_{s}^{2} \Delta t$, where $c_{s}=(1 / \sqrt{3})(\Delta x / \Delta t)$ is the so-called "lattice speed of sound". The equilibrium distribution function $f_{i}^{\mathrm{eq}}(\mathbf{x}, t)$ corresponds to the series expansion of Maxwell-Boltzmann distribution for small Mach numbers. The additional term here $\tilde{\Omega}_{i}(\mathbf{x}, t)$ is caused by the coupling forces $\mathbf{F}(\mathbf{x}, t)$ between the blood cells and the fluid (and/or any external force field) 203.

The immersed boundary method (IB) is a coupling method applicable for the description of dynamics of cell membranes immersed in blood plasma 202. This is however, not the only application, as a number of works use IB for the granular media and suspension flows simulations. A great advantage of IBM, is that the fluid and the immersed structure (cell) do not need to conform via their meshed or grids, thus there is no need for remeshing. The central idea of IB method is the coupling between a viscous incompressible fluid and a 
number of moving deformable objects in contact with that fluid. Fluid drag and normal stresses agitate motion of the objects and objects (blood cells) in their turn influence the motion of the fluid. The elastic cell membrane is described in Lagrangian terms: Langrangian surface points (or surface particles) which constitute the membrane mesh, exert a force to the fluid, and the fluid advects the surface particles to their new positions by interpolating the fluid velocity. The surface particle belonging to the cell membrane $\mathbf{X}_{i}(t)$ spreads the total force $\mathbf{F}_{i}(t)$ exerted on it to the closest fluid-grid points $\mathbf{x}$ :

$$
\mathbf{F}(\mathbf{x}, t)=\sum_{i} \mathbf{F}_{i}(t) \delta\left(\mathbf{x}-\mathbf{X}_{i}(t)\right)
$$

with interpolation kernel (discrete Dirac delta function) $\delta\left(\mathbf{x}-\mathbf{X}_{i}(t)\right.$ ). This force field $\mathbf{F}(\mathbf{x}, t)$ is exerted on the fluid via $\tilde{\Omega}$ term in Eq. 53 (see References 203] and 204 for details). The membrane particles gain increment to their coordinates

$$
\mathbf{x}_{i}(t+\Delta t)=\mathbf{x}_{i}(t)+\mathbf{u}_{i}(t+\Delta t) \Delta t
$$

as they are advected by the nearby fluid with the velocity

$$
\mathbf{u}_{i}(t+\Delta t)=\sum_{\mathbf{x}} \mathbf{u}_{i}(\mathbf{x}, t) \delta\left(\mathbf{x}-\mathbf{X}_{i}(t)\right)
$$

\subsubsection{Efficiency, stability and convergence on example of LBM-IB scheme}

The LBM is stable for small Mach numbers $M a \ll 1$, so that any velocities in model system should be much lower than lattice speed of sound $U \ll c_{s}=$ $(1 / \sqrt{3}) \Delta x / \Delta t$, where $\Delta x$ is a Lattice unit and $\Delta t$ is the time-step for iterations [135. Convergence of the solution to the incompressible Navier-Stokes equations for a chosen Reynolds number is obtained by letting the lattice Mach number $U / c_{s}$ become small enough to remove compressibility effects. In terms of lattice parameters the convergence for a given Reynolds number could be achieved if the following condition is satisfied:

$$
\frac{\sqrt{3} \Delta t U}{\Delta x} \ll 1,
$$

which implies that either decreasing time step $\Delta t$ or mesh refinement $\Delta x$ provide convergence. While in the latter case the efficacy may be maintained by the 
proportional increase of processors, in the first case the increase of the number of iterations is needed to reach the same flow evolution time. For a given Reynolds number $R e$ one can reformulate this conditions in the following way:

$$
\Delta x \ll \frac{\sqrt{3} L}{\operatorname{Re}(\tau-0.5)},
$$

where $L$ is the spacial scale of the system.

Taking into account that in microvascular $(L<100 \mu \mathrm{m})$ blood flows $R e \leq$

forces, bending forces, synchronization between subdomains, etc.) is the most consuming part of these hybrid methods, followed by the Immersed Boundary membrane dynamics [201]. The Lattice Boltzmann fluid (plasma) dynamics is 
still the fastest part of such a hybrid model, in case that the model parameters

\subsubsection{Alternative approaches}

Several variations exist, like the substitution of LBM with conventional Navier-Stokes [205, 178] and/or usage of different fluid-cell coupling [178, 206]. For example, Moving Particle Semi-Implicit method [207, 208] based on NavierStokes equation solver combined with Lagrangian particle dynamics, which was quite successful in describing motion of malaria-infected RBCs in narrow vessels.

Smoothed Dissipative Particle Dynamics (SDPD) 209 method has been used by [210] to study WBC margination in whole blood flows. This method combines two frequently employed approaches: smoothed particle hydrodynamics and fluctuating nature of dissipative particle dynamics (DPD). The approach to blood simulation was widely used: blood cells were represented by a network membrane model, using triangulated surfaces with curvature and stretching elasticity, the blood cells were suspended in a Newtonian fluid (plasma), which is represented by a collection of SDPD particles. Cell membranes were coupled the LBM-IB method describe above.

Another set of publications took advantage of Boundary Integral Method [211, 174, 146] to solve the boundary integral equations for Stokes flow. These equations express conservation of momentum as a relation between the velocity of an object (cell) and the applied force. This approach is a common numerical computational method of solving linear partial differential equations that have to be formulated as integral equations (i.e. in boundary integral form). It is only applicable to problems for which Green's functions can be calculated. The Green's functions, or fundamental solutions, are often problematic to integrate, especially for complex geometries. Nevertheless, Boundary Integral Method (BIM) works by constructing a "mesh" over the modelled surface, rather than in the whole space, therefore, it is often more efficient than finite elements method. 


\subsubsection{Parallelism and related issues}

Simulations of dense blood-like suspensions require considerable computational resources. Parallel computations could be advantageous in this class of problems. However, the software, in terms of algorithms, data structures and parallelism, is complex when dealing with hybrid models with a number of modules of distinctive efficiency. A number of new techniques had to be developed before the large-scale cell-level simulations of blood flows could be performed. For instance, complexity of a problem increases rapidly when dealing with boundary conditions, moving objects, and suspended particles, and parallelism may not be straight-forward in these cases due to synchronization between nodes and processors. New algorithms are required, as well as the control of data structures and streams, efficient scaling and domain decomposition. For instance, finding neighbouring particles for cell-cell interactions can be a time consuming task and cell-lists (analogue to Verlet-lists) can reduce potential complexity from $O\left(N^{2}\right)$ to $O(N)$, which is crucial when the number of particles $N$ becomes large.

Domain decomposition has its limits. This means that one cannot just extensively overcome the complexity by increasing the number of MPI-nodes carrying out the simulation. The parallelism usually assumes the domain-decomposition of the fluid field, and the subdivision of cell-membrane particles follows this partition. However, as the membrane particles interact with each other forming a consolidated object - a cell - the communications have to be set and controlled between subdomains, when a cell travels from one subdomain to another. For these reasons, envelopes were proposed 201. Envelopes are the boundary nodes communicating in the domain-decomposition approach, also commonly referred to as ghost nodes. This allows for a direct identification of the particles that belong to the envelopes and communicate them to the neighbouring subdomains. Optimizing communication is an important aspect of the implementation.

Another issue is that different modules of a software have different efficacy. The most demanding is the membrane-mechanics module, while the fastest is 
fluid module (especially if implemented via Lattice Boltzmann approach) 201].

Overall, cell-based models are detailed and can help to understand the importance of cell-cell interactions and effects on the subcellular scale, yet the computational difficulties limit their applicability nowadays to short timescales (seconds, tens of seconds), whence thrombosis and hemostasis processes usually take tens of minutes. The search for new soft- and hard-ware solutions is open,

1325 however. New advances in computational sciences and computing technologies (FPGA, GPU, etc.) are very promising.

\section{Perspectives and future challenges}

Mathematical modelling of thrombus formation has achieved remarkable sophistication. In the previous sections we have stated the milestones in the construction of models in the field of haemostasis. These works investigate subsystems within thrombus formation (such as thrombin generation) as well as the establishment of links between different subsystems, e.g. plasma clotting, signalling, platelet aggregation and activation, fluid dynamics, chemical reactions, etc. These models allow us to take note of the influence of these links. For example, we can notice the influence of hydrodynamic flows which are crucial for all scales present in the system from the biochemical (protein) level to the macroscopic one. The modelling is also aiding in the establishment of similarities between the behaviours of plasma clotting and platelet aggregates under flow.

The ideal, state-of-the-art model would comprise a full mathematical description of thrombus formation in flow that included the reactions of the coagulation cascade, interactions inside and between the cells in blood (platelets, red blood cells and ethrocytes) as well as haemodynamic considerations. This would be highly complex model and its construction is not just a theoretical challenge but a biological one, especially where insufficient knowledge is leading to model uncertainty, though of course modelling attempts can also aid with validating biological hypothesis surrounding this uncertainty. But this is worth 
attempting, the outcome of such an approach could be very important for both fundamental science and practical medicine.

The development of any theoretical model of a biological system such as a thrombus has the dual aim of increasing our understanding of the system in question and the ability to make accurate predictions. Theoretical models of thrombus formation are currently fulfilling the first of these aims and increasing attempts are being made at producing models that are accurate and/or have their accuracy (or inaccuracies) quantified. The ability to make these accurate, and indeed quantitative, predictions relies on the accuracy of the model's parameter values. But, the vast majority of a model's parameters are difficult or impossible to measure experimentally, for example the immunoassays utilised to describe intracellular changes are limited by the availability and quality of antibodies, therefore modellers have traditionally relied on parameter values that have been gleaned from literature 212. These values often based on experiments carried out under different conditions or even within different cell types introduce uncertainties and errors into model predictions. Fortunately this is an area of active research [13, 213, 214 and new theoretical techniques, high throughput experimental data and computational power are increasing our ability to infer model parameters from experimental data and therefore develop more reliable models. But this remains still a formidable challenge where spatial, stochastic and multiscale events are involved. Addressing this requiring not only new mathematical and computational techniques but a community wide cross-disciplinary effort to openly share data and protocols so that parameter inference can occur across all scales.

As discussed above, haemostasis is a complex systemic phenomenon based on a subtle equilibrium between various pro- and antithrombotic factors. Its malfunctioning can lead to numerous bleeding disorders or thrombotic events 1375 and represents major direct, or indeed indirect, causes of mortality and morbidity. One of the most dangerous and common among these is deep venous thrombosis (DVT), a blood clotting disorder that occurs when excessive blood clotting occurs inside deep veins. It can provoke embolisms where a part of the 
clot breaks off and subsequently blocks blood circulation in smaller vessels.

The risk factors for developing deep venous thrombosis are numerous and include obesity, cancer, injury and slow blood flow due to the lack of physical activity. Recurrent thrombi can be prevented by controlling these risk factors or by the use of anticoagulant drugs. There are several anticoagulant drugs that target the coagulation cascade at different points. Some new anticoagulants target single points ,such as factor Xa or thrombin [215. Others like heparin increase the activity of antithrombin [216]. Another important type of anticoagulant drugs is vitamin $\mathrm{K}$ antagonist (AVK) drugs such as warfarin. It acts by reducing vitamin K-dependent factors modification in the liver [217. As a result, the functionalities of the key blood clotting factors such as prothrombin and factors IX and $\mathrm{X}$ are decreased.

Warfarin treatments are monitored by blood tests for the International Normalized Ratio (INR). This is an in vitro assay based on the measurement of the prothrombin time (PT). This characterizes the time interval necessary to convert prothrombin into thrombin under specific experimental conditions. Depending on the measured value of INR, the administrated dose of warfarin is adjusted in order to reach a therapeutic range. In DVT, the targeted level of INR is between 2.0 and 3.0 [218]. A lower than 2.0 value of INR indicates the predisposition of the patient to develop recurrent thrombosis while a higher than 3.0 value signifies a high risk of bleeding. The difference between the experimental settings under which the INR is measured and in vivo physiological conditions can result in cardio-vascular events even for a normal INR level. These tests are flawed as they do not consider the effect of blood flow and the direct inhibition of thrombin by antithrombin [110].

One of the main issues in the treatment of thrombosis is the individual 1405 selection of the medication and protocol of its administration on the basis of personalized patient specific data. This is an area where mathematical modeling can be extremely useful, but at present it is obviously not used enough. This is due not only to the difficulties in constructing theoretical models but also because systems available for obtaining and processing individual donor data 


\section{Acknowledgements}

AVB acknowledges support from Russian Foundation for Basic Research (1631-60061-mol-a-dk) and the Stipend of the President of Russian Federation (SP2427.2015.4). MAP acknowledges support from Russian Federation President 1435 Grant for Young Doctors of Science (MD-229.2017.4), from Russian Foundation for Basic Research Grant (KOMFI 17-00-00140) and the grant from the endowment foundation "Doctors, innovations, science for children". VV was partially supported by the Ministry of Education and Science of the Russian Federation 
(the agreement number 02.a03.21.0008).

\section{References}

[1] A. Shibeko, M. Panteleev, Untangling the complexity of blood coagulation network: use of computational modelling in pharmacology and diagnostics., Brief Bioinform. 17 (2016) 429-439.

[2] A. P. Bye, A. J. Unsworth, J. M. Gibbins, Platelet signaling: a complex interplay between inhibitory and activatory networks, Journal of Thrombosis and Haemostasis 14 (5) (2016) 918-930. doi:10.1111/jth.13302.

[3] L. Holbrook, G. Sandhar, P. Sasikumar, M. Schenk, A. Stainer, K. Sahli, G. Flora, A. Bicknell, J. Gibbins, A humanized monoclonal antibody that inhibits platelet-surface erp72 reveals a role for erp72 in thrombosis, Journal of Thrombosis and Haemostasisdoi:10.1111/jth.13878

[4] N. N. Topalov, A. O. Yakimenko, M. Canault, E. O. Artemenko, N. V. Zakharova, A. A. Abaeva, M. Loosveld, F. I. Ataullakhanov, A. T. Nurden, M.-C. Alessi, M. A. Panteleev, Two types of procoagulant platelets are formed upon physiological activation and are controlled by integrin $\alpha_{I I b} \beta_{3}$, Arteriosclerosis, Thrombosis, and Vascular Biology 32 (2012) 2475-2483. doi:10.1161/ATVBAHA.112.253765.

[5] S. Vaiyapuria, G. Flora, J. Gibbins, Gap junctions and connexin hemichannels in the regulation of haemostasis and thrombosis, Biochem. Soc. Trans. 43 (3) (2015) 489-94. doi:10.1042/BST20150055.

[6] A. Yakimenko, F. Verholomova, Y. Kotova, F. Ataullakhanov, M. Panteleev, Identification of different proaggregatory abilities of activated platelet subpopulations., Biophys J. 102 (2012) 2261-2269. doi:10.1016/ j.bpj.2012.04.004 
[7] M. Panteleev, V. Zarnitsina, F. Ataullakhanov, Tissue factor pathway inhibitor: a possible mechanism of action., Eur J Biochem. 269 (8) (2002) 2016-2031. doi:10.1046/j.1432-1033.2002.02818.x.

[8] M. Panteleev, N. Ananyeva, N. Greco, F. Ataullakhanov, E. Saenko, Factor viiia regulates substrate delivery to the intrinsic factor $\mathrm{x}$-activating complex, FEBS Journal 273 (2006) 374-387. doi:10.1111/j.1742-4658. $2005.05070 \cdot x$

[9] N. Bessonov, E. Babushkina, S. F. Golovashchenko, A. Tosenberger, F. Ataullakhanov, M. Panteleev, A. Tokarev, V. Volpert, Numerical modelling of cell distribution in blood flow, Math. Model. Nat. Phenom. 9 (6) (2014) 69-84.

[10] A. Tosenberger, F. Ataullakhanov, N. Bessonov, M. Panteleev, A. Tokarev, V. Volpert, Modelling of thrombus growth in flow with a dpd-pde method, Journal of Theoretical Biology 337 (2013) 30-41.

[11] A. V. Belyaev, M. A. Panteleev, F. I. Ataullakhanov, Threshold of microvascular occlusion: Injury size defines the thrombosis scenario, Biophysical Journal 109 (2015) 450-456.

[12] A. Tosenberger, F. Ataullakhanov, N. Bessonov, M. Panteleev, A. Tokarev, V. Volpert, Modelling of platelet-fibrin clot formation in flow with a dpd-pde method, Journal of Mathematical Biology 72 (3) (2016) 649-681.

[13] J. Dunster, F. Mazet, M. Fry, J. Gibbins, M. Tindall, Regulation of early steps of gpvi signal transduction by phosphatases: A systems biology approach., PLoS Comput Biol. 11 (2015) e1004589.

[14] A. Sveshnikova, F. Ataullakhanov, M. Panteleev, Compartmentalized calcium signaling triggers subpopulation formation upon platelet activation through par1., Mol Biosyst. 11 (2015) 1052-1060. 
[15] A. Sveshnikova, A. Balatskiy, A. Demianova, T. Shepelyuk, S. Shakhidzhanov, M. Balatskaya, A. Pichugin, F. Ataullakhanov, M. Panteleev, Systems biology insights into the meaning of the platelet's dual-receptor thrombin signaling., J. Thromb. Haemost. 14 (2016) 2045-2057.

[16] M. Gissel, C. Holmes, R. Branda, L. Ferris, K. Brummel-Ziedins, Plasma composition distribution in cancer: Discrimination by thrombin and factor xa generation., Thromb Res. 146 (2016) 56-58.

[17] Y. Lu, M. Lee, S. Zhu, T. Sinno, S. Diamond, Multiscale simulation of thrombus growth and vessel occlusion triggered by collagen/tissue factor using a data-driven model of combinatorial platelet signalling., Math Med Biol doi:10.1093/imammb/dqw015.

[18] P. Aarts, S. van den Broek, G. Prins, G. Kuiken, J. Sixma, R. Heethaar, Blood platelets are concentrated near the wall and red blood cells, in the center in flowing blood., Arteriosclerosis 8 (1988) 819-824.

[19] N. Bessonov, E. Babushkina, S. Golovashchenko, A. Tosenberger, F. Ataullakhanov, M. Panteleev, A. Tokarev, V. Volpert, Numerical simulation of blood flows with non-uniform distribution of erythrocytes and platelets., Russian journal of numerical analysis and mathematical modelling 28 (2013) 443-458.

[20] A. Tokarev, A. Butylin, F. Ataullakhanov, Platelet adhesion from shear blood flow is controlled by near-wall rebounding collisions with erythrocytes., Biophys J. 100 (2011) 799-808.

[21] A. Tokarev, A. Butylin, E. Ermakova, E. Shnol, G. Panasenko, 1515 F. Ataullakhanov, Finite platelet size could be responsible for platelet margination effect., Biophys J. 101 (2011) 1835-1843.

[22] B. Walton, M. Lehmann, T. Skorczewski, L. Holle, J. Beckman, J. Cribb, M. Mooberry, A. Wufsus, B. Cooley, J. Homeister, R. Pawlinski, M. Falvo, 
N. Key, A. Fogelson, K. Neeves, A. Wolberg, Elevated hematocrit enhances platelet accumulation following vascular injury., Blood 129 (2017) $2537-2546$.

[23] F. Balabin, A. Sveshnikova, Computational biology analysis of platelet signaling reveals roles of feedbacks through phospholipase $\mathrm{c}$ and inositol 1,4,5-trisphosphate 3-kinase in controlling amplitude and duration of calcium oscillations, Math Biosci. 276 (2016) 67-74.

[24] L. Brass, S. Diamond, Transport physics and biorheology in the setting of hemostasis and thrombosis., J Thromb Haemost. 14 (2016) 906-917.

[25] A. Shibeko, E. Lobanova, M. Panteleev, F. Ataullakhanov, Blood flow controls coagulation onset via the positive feedback of factor vii activation by factor xa., BMC Syst Biol. 4 (2010) 5.

[26] M. Panteleev, N. Dashkevich, F. Ataullakhanov, Hemostasis and thrombosis beyond biochemistry: roles of geometry, flow and diffusion., Thromb Res. 136 (2015) 699-711.

[27] K. Leiderman, A. Fogelson, The influence of hindered transport on the development of platelet thrombi under flow., Thromb Res. 75 (2013) 12551283.

[28] M. Kamocka, J. Mu, X. Liu, N. Chen, A. Zollman, B. Sturonas-Brown, K. Dunn, Z. Xu, D. Chen, M. Alber, E. Rosen, Two-photon intravital imaging of thrombus development., J Biomed Opt. 15 (2010) 016020.

[29] E. Kenne, K. Nickel, A. Long, T. Fuchs, E. Stavrou, F. Stahl, T. Renne, Factor xii: a novel target for safe prevention of thrombosis and inflammation., J Intern Med 278 (2015) 571-585.

[30] F. Muller, N. Mutch, W. Schenk, S. Smith, L. Esterl, H. Spronk, S. Schmidbauer, W. Gahl, J. Morrissey, T. Renne, Platelet polyphosphates are proinflammatory and procoagulant mediators in vivo., Cell 139 (2009) $1143-1156$. 
[31] N. Zakharova, E. Artemenko, N. Podoplelova, A. Sveshnikova, I. Demina, F. Ataullakhanov, M. Panteleev, Platelet surface-associated activation and secretion-mediated inhibition of coagulation factor xii., PLoS One 10 (2) (2015) e0116665.

[32] A. Abaeva, M. Canault, Y. Kotova, S. Obydennyy, A. Yakimenko, N. Podoplelova, V. Kolyadko, H. Chambost, A. Mazurov, F. Ataullakhanov, A. Nurden, M. Alessi, M. Panteleev, Procoagulant platelets form an alpha-granule protein-covered "cap" on their surface that promotes their attachment to aggregates., J Biol Chem. 288 (2013) 29621-29632.

[33] N. Podoplelova, A. Sveshnikova, Y. Kotova, A. Eckly, N. Receveur, D. Nechipurenko, S. Obydennyi, I. Kireev, C. Gachet, F. Ataullakhanov, P. Mangin, M. Panteleev, Coagulation factors bound to procoagulant platelets concentrate in cap structures to promote clotting., Blood 128 (2016) 1745-1755.

[34] N. Podoplelova, A. Sveshnikova, J. Kurasawa, A. Sarafanov, H. Chambost, S. Vasil'ev, I. Demina, F. Ataullakhanov, M. Alessi, M. Panteleev, Hysteresis-like binding of coagulation factors $\mathrm{x} / \mathrm{xa}$ to procoagulant activated platelets and phospholipids results from multistep association and membrane-dependent multimerization., Biochim Biophys Acta 1858 (2016) 1216-1227.

[35] N. Arraud, R. Linares, S. Tan, C. Gounou, J. Pasquet, S. Mornet, A. Brisson, Extracellular vesicles from blood plasma: determination of their morphology, size, phenotype and concentration., J Thromb Haemost. 12 (2014) 614-627.

[36] E. Lipets, O. Vlasova, E. Urnova, O. Margolin, A. Soloveva, O. Ostapushchenko, J. Andersen, F. Ataullakhanov, M. Panteleev, Circulating contact-pathway-activating microparticles together with factors ixa and 
xia induce spontaneous clotting in plasma of hematology and cardiologic patients, PLoS One 9 (2014) e87692.

[37] E. Sinauridze, D. Kireev, N. Popenko, A. Pichugin, M. Panteleev, O. Krymskaya, F. Ataullakhanov, Platelet microparticle membranes have 50- to 100-fold higher specific procoagulant activity than activated platelets, Thromb Haemost. 97 (2007) 425-434.

[38] X. Liang, I. Chernysh, P. Purohit, J. Weisel, Phase transitions during compression and decompression of clots from platelet-poor plasma, plateletrich plasma and whole blood, Acta Biomater. 60 (2017) 275-290.

[39] M. Panteleev, A. Balandina, E. Lipets, M. Ovanesov, F. Ataullakhanov, Task-oriented modular decomposition of biological networks: trigger mechanism in blood coagulation., Biophys J. 98 (2010) 1751-1761.

[40] M. Panteleev, M. Ovanesov, D. Kireev, A. Shibeko, E. Sinauridze, N. Ananyeva, A. Butylin, E. Saenko, F. Ataullakhanov, Spatial propagation and localization of blood coagulation are regulated by intrinsic and protein C pathways, respectively., Biophys J. 90 (5) (2006) 1489-1500.

[41] J. Dunster, J. King, Mathematical modelling of thrombin generation: asymptotic analysis and pathway characterization., IMA Journal of Applied Mathematics. 82 (2017) 60-96.

[42] C. Danforth, T. Orfeo, S. Everse, K. Mann, K. Brummel-Ziedins, Defining the boundaries of normal thrombin generation: investigations into hemostasis, PloS ONE. 7 (2012) e30385.

[43] A. Mitrophanov, J. Reifman, Kinetic modeling sheds light on the mode of action of recombinant factor viia on thrombin generation, Thrombosis research 128 (2011) 381-390.

[44] M. Hockin, K. Jones, S. Everse, K. Mann, A model for the stoichiometric regulation of blood coagulation, Journal of Biological Chemistry 277 (2002) 18322-18333. 
[45] N. Dashkevich, M. Ovanesov, A. Balandina, S. Karamzin, P. Shestakov, N. Soshitova, A. Tokarev, M. Panteleev, F. Ataullakhanov, Thrombin activity propagates in space during blood coagulation as an excitation wave, Biophys J. 103 (2012) 2233-2240.

[46] L. Parunov, O. Fadeeva, A. Balandina, N. Soshitova, K. Kopylov, M. Kumskova, J. Gilbert, R. Schaub, K. McGinness, F. Ataullakhanov, M. Panteleev, Improvement of spatial fibrin formation by the anti-tfpi aptamer bax499: changing clot size by targeting extrinsic pathway initiation, J Thromb Haemost. 9 (2011) 1825-1834.

[47] A. Tripodi, Thrombin generation assay and its application in the clinical laboratory, Clin. Chem. 62 (5) (2016) 699-707. doi:10.1373/clinchem. 2015.248625

[48] I. Tarandovskiy, A. Balandina, K. Kopylov, N. Konyashina, M. Kumskova, M. Panteleev, F. Ataullakhanov, Investigation of the phenotype heterogeneity in severe hemophilia a using thromboelastography, thrombin generation, and thrombodynamics, Thrombosis Research 131 (6) (2013) e274-e280. doi:10.1016/j.thromres.2013.04.004.

[49] G. Pike, A. Cumming, C. Hay, P. Bolton-Maggs, J. Burthem, Sample conditions determine the ability of thrombin generation parameters to identify bleeding phenotype in fxi deficiency., Blood 126 (2015) 397-405.

[50] V. N. Kolyadko, S. V. Lushchekina, T. A. Vuimo, S. S. Surov, R. A. Ovsepyan, V. A. Korneeva, I. I. Vorobiev, N. A. Orlova, L. Minakhin, K. Kuznedelov, K. V. Severinov, F. I. Ataullakhanov, M. A. Panteleev., New infestin-4 mutants with increased selectivity against factor xiia., PLoS One 10 (12) (2015) e0144940.

[51] N. Zakharova, E. Artemenko, N. Podoplelova, A. Sveshnikova, I. Demina, F. Ataullakhanov, M. Panteleev, Platelet surface-associated activation and secretion-mediated inhibition of coagulation factor xii., PLoS One 10 (2) (2015) e0116665. 
[52] A. Matafonov, S. Sarilla, M. Sun, J. Sheehan, V. Serebrov, I. Verhamme, D. Gailani, Activation of factor xi by products of prothrombin activation., Blood 118 (2011) 437-445.

[53] S. Butenas, K. Mann, Blood coagulation, Biochem. (Moscow) 67 (31) (2002) 3-12.

[54] T. Orfeo, S. Butenas, K. Brummel-Ziedins, K. Mann, The tissue factor requirement in blood coagulation, J. Biol. Chem. 280 (52) (2005) 4288742896.

[55] H. Hemker, Thrombin generation, an essential step in haemostasis and thrombosis, Haemost. Thromb. 3 (1993) 477-491.

[56] H. Hemker, S. Beguin, Thrombin generation in plasma: its assessment via the endogenous thrombin potential, Thromb. Haemost. 74 (1) (1995) $134-138$.

[57] T. Orfeo, K. Brummel-Ziedins, M. Gissel, S. Butenas, K. Mann, The nature of the stable blood clot procoagulant activities, J. Biol. Chem. 283 (15) (2008) 9776-9786.

[58] D. Gailani, G. Broze, Factor xi activation in a revised model of blood coagulation, Science 253 (5022) (1991) 909-912.

[59] S. Bungay, P. Gentry, R. Gentry, A mathematical model of lipid-mediated thrombin generation, Mathematical Medicine and Biology 20 (1) (2003) $105-29$.

[60] K. C. Jones, K. G. Mann, A model for the tissue factor pathway to thrombin, Journal of Biological Chemistry 269 (37) (1994) 23367-73.

[61] M. Hockin, A model for the stoichiometric regulation of blood coagulation, Journal of Biological Chemistry 277 (21) (2002) 18322-33.

[62] M. Khanin, D. Rakov, A. Kogan, Mathematical model for the blood coagulation prothrombin time test, Thrombosis research 89 (5) (1998) 227-32. 
[63] C. Xu, X. H. Xu, Y. Zeng, Y. W. Chen, Simulation of a mathemat-

[67] D. Zhu, Mathematical modeling of blood coagulation cascade: kinetics of intrinsic and extrinsic pathways in normal and deficient conditions, Blood

[69] D. Luan, M. Zai, J. Varner, Computationally derived points of fragility of a human cascade are consistent with current therapeutic strategies, PLoS [ ical model of the role of the tfpi in the extrinsic pathway of coagulation, Computers in Biology and Medicine 35 (5) (2005) 435-45. doi: 10.1016/j.compbiomed.2004.02.007.

[64] L. Braescu, M. Leretter, T. George, New direct inhibitors and their computed effect on the dynamics of thrombin formation in blood coagulation, in: T. F. George (Ed.), Computational Studies of New Materials II, World Scientific, 2011, pp. 173-190. doi:10.3389/fphys.2012.00266.

[65] K. Tyurin, M. Khanin, Hemostasis as an optimal system, Mathematical Biosciences 204 (2) (2006) 167-4.

[66] V. Zarnitsina, A. Pokhilko, F. Ataullakhanov, A mathematical model for the spatio-temporal dynamics of intrinsic pathway of blood coagulation. i. the model description, Thrombosis Research 84 (4) (1996) 225-36. doi: $10.1016 / \mathrm{j} . \mathrm{bpj} .2009 .12 .4331$.

Coagulation and Fibrinolysis 18 (7) (2007) 637-46. doi:10.1097/MBC. 0b013e3282a167bb

[68] M. Chatterjee, W. Denney, H. Jing, S. Diamond, Systems biology of coagulation initiation: Kinetics of thrombin generation in resting and activated human blood., PLoS Computational Biology 6 (9) (2010) 1-24. doi:10.1371/journal.pcbi.1000950.

Computational Biology 3 (7) (2007) e142. doi:10.1371/journal.pcbi. 0030142 .

[70] M. Panteleev, A. Balandina, E. Lipets, M. Ovanesov, F. Ataullakhanov, Task-oriented modular decomposition of biological networks: Trigger 
mechanism in blood coagulation, Biophysical Journal 98 (9) (2010) 175161.

[71] Z. Xu, J. Lioi, J. Mu, M. Kamocka, X. Liu, D. Chen, E. Rosen, M. Alber,

[74] A. Kuharsky, A. Fogelson, Surface-mediated control of blood coagulation: the role of binding site densities and platelet deposition, Biophysical journal 80 (3) (2001) 1050-1074.

[75] S. Jordan, E. Chaikof, Simulated surface-induced thrombin generation in

[76] D. LaCroix, A reduced equation mathematical model for blood coagulation and fibrinolysis in quiescent plasma, The International Journal of Structural Changes in Solids 4 (2012) 23-35.

[77] T. Wajima, G. Isbister, S. Duffull, A comprehensive model for the humoral coagulation network in humans, Clinical Pharmacology \& Therapeutics 86 (3) (2009) 290-8.

[78] G. Willems, T. Lindhout, W. Hermens, H. Hemker, Simulation model for thrombin generation in plasma, Haemostasis 21 (1991) 197-207. 
[79] S. Woodle, A. Shibeko, T. Lee, M. Ovanesov, Determining the impact of instrument variation and automated software algorithms on the tgt in hemophilia and normalized plasma, Thrombosis Research 132 (3) (2013) 374-380. doi:10.1016/j.thromres.2013.07.018

[80] Y. Liang, S. Woodle, A. Shibeko, T. Lee, M. Ovanesov, Correction of microplate location effects improves performance of the thrombin generation test, Thrombosis Journal 11 (1) (2013) 12. doi:10.1186/ 1477-9560-11-12.

[81] F. I. Ataullakhanov, G. T. Guria, V. I. Sarbash, R. I. Volkova, Spatiotemporal dynamics of clotting and pattern formation in human blood, Biochimica et biophysica acta 1425 (1998) 453-468.

[82] Y. V. Krasotkina, E. I. Sinauridze, F. Ataullakhanov, Spatiotemporal dynamics of fibrin formation and spreading of active thrombin entering non-recalcified plasma by diffusion, Biochimica et Biophysica Acta (BBA)General Subjects 1474 (3) (2000) 337-345.

[83] P. Chelle, C. Morin, A. Montmartin, M. Piot, M. Cournil, B. TardyPoncet, Evaluation of in silico models of thrombin generation using experimental data from healthy and haemophilic subjects, In press.

[84] P. Chelle, C. Morin, A. Montmartin, M. Piot, M. C. andBrigitte TardyPoncet, Subject-specific identification of kinetic parameters in the thrombin generation modelling, In press.

[85] V. I. Zarnitsina, A. V. Pokhilko, F. I. Ataullakhanov, A mathematical model for the spatio-temporal dynamics of intrinsic pathway of blood coagulation. ii. results, Thrombosis Research 84 (5) (1996) 333-344.

[86] V. I. Zarnitsina, F. I. Ataullakhanov, A. I. Lobanov, O. L. Morozova, Dynamics of spatially nonuniform patterning in the model of blood coagulation, Chaos 11 (2001) 57. 
[87] A. Tokarev, Y. Krasotkina, M. Ovanesov, M. Panteleev, M. Azhigirova, Spatial dynamics of contact-activated fibrin clot formation in vitro and in silico in haemophilia b: Effects of severity and ahemphil b treatment, Math. Model. Nat. Phenom. 1474 (2006) 124.

[88] E. A. Pogorelova, A. I. Lobanov, Influence of enzymatic reactions on blood coagulation autowave, Biophysics 59 (1) (2014) 110-118.

[89] K. Guria, G. Guria, Spatial aspects of blood coagulation: Two decades of research on the self-sustained traveling wave of thrombin, Thrombosis Research 135 (3) (2015) 423-433.

[90] T. Galochkina, H. Ouzzane, A. Bouchnita, V. Volpert, Traveling wave solutions in the mathematical model of blood coagulation, Applicable Analysis 96 (16) (2017) 2891-2905.

[91] T. Galochkina, A. Bouchnita, P. Kurbatova, V. Volpert, Reactiondiffusion waves of blood coagulation, Mathematical Biosciences 288 (2017) 130-139.

[92] A. Volpert, V. Volpert, V. Volpert., Traveling wave solutions of parabolic systems, American Mathematical Society, Providence, 1994.

[93] V. Volpert, Elliptic partial differential equations. Volume 2: Reactiondiffusion equations, Birkhäuser, 2014.

${ }_{1760}$ [94] M. Marion, V. Volpert, Existence of pulses for the system of competition of a species, Journal of Dynamics and Differential Equations (2017) 1-18doi: $10.1007 / \mathrm{s} 10884-017-9582-6$.

[95] M. Marion, T. Galochkina, V. Volpert, Initiation of reaction-diffusion waves of blood coagulation, Physica D: Nonlinear PhenomenaIn press.

1765 [96] A. Bouchnita, A. Tosenberger, V. Volpert, On the regimes of blood coagulation, Applied Mathematics Letters 51 (3) (2016) 74-79. 
[97] A. Bouchnita, T. Galochkina, P. Kurbatova, P. Nony, V. Volpert, Conditions of microvessel occlusion for blood coagulation in flow, International Journal for Numerical Methods in Biomedical Engineering 33 (9) (2017) e2850. doi:10.1002/cnm.2850.

[98] R. Guy, A. Fogelson, J. Keener, Fibrin gel formation in a shear flow, Math. Med. Biol. 24 (2007) 111-130.

[99] L. M. Haynes, Y. Dubief, K. Mann, Membrane binding events in the initiation and propagation phases of tissue factor-initiated zymogen activation under flow, Journal of Biological Chemistry 287 (8) (2012) 5225-5234.

[100] A. I. Lobanov, T. K. Starozhilova, The effect of convective flows on blood coagulation processes, Pathophysiology of haemostasis and thrombosis $34(2-3)$ (2005) 121-134.

[101] A. L. Fogelson, N. Tania, Coagulation under flow: the influence of flowmediated transport on the initiation and inhibition of coagulation, Pathophysiology of haemostasis and thrombosis 34 (2-3) (2005) 91-108.

[102] S. W. Jordan, E. L. Chaikof, Simulated surface-induced thrombin generation in a flow field, Biophysical journal 101 (2) (2011) 276-286.

[103] V. Govindarajan, V. Rakesh, J. Reifman, A. Y. Mitrophanov, Computational study of thrombus formation and clotting factor effects under venous flow conditions, Biophysical journal 110 (8) (2016) 1869-1885.

[104] S. Gir, S. Slack, V. Turito, A numerical analysis of factor x activation in the presence of tissue factor-factor viia complex in a flow reactor, Annals of Biomedical Engineering 24 (8) (1996) 394-399.

1790

[105] A. Sequeira, R. Santos, T. Bodnar, Blood coagulation dynamics: mathematical modeling and stability results, Mathematical biosciences and engineering 8 (2) (2011) 425-443. 
[106] T. Bodnar, A. Sequeira, Numerical simulation of the coagulation dynamics of blood, Computational and Mathematical Methods in Medicine 9 (2) (2008) 83-104.

[107] A. Sequeira, T. Bodnar, Blood coagulation simulations using a viscoelastic model, Mathematical Modelling of Natural Phenomena 9 (6) (2014) 3445.

[108] A. Wufsus, N. Macera, K. Neeves, The hydraulic permeability of blood clots as a function of fibrin and platelet density, Biophys J 104 (8) (2013) 1812-23. doi:10.1016/j.bpj.2013.02.055

[109] A. Bouchnita, T. Galochkina, V. Volpert., Influence of antithrombin on the regimes of blood coagulation: insights from the mathematical model, Acta Biotheoretica 64 (4) (2016) 327-342.

[110] A. Bouchnita, K. Bouzaachane, T. Galochkina, P. Kurbatova, P. Nony, V. Volpert, An individualized blood coagulation model to predict inr therapeutic range during warfarin treatment, Math. Model, Nat. Phenom 11 (6) (2016) 28-44.

[111] A. Bouchnita, P. Miossec, A. Tosenberger, V. Volpert, Modeling of the effects of il-17 and tnf-a on endothelial cells and thrombus growth, Comptes rendus Biologiesdoi:10.1016/j.crvi.2017.10.002

[112] S. P. Jackson, W. S. Nesbitt, E. Westein, Dynamics of platelet thrombus formation, Journal of Thrombosis and Haemostasis 7 (1) (2009) 17-20.

[113] B. Furie, B. C. Furie, Thrombus formation in vivo, J. Clin. Invest. 115 1815 (2005) 3355-3362.

[114] T. J. Stalker, E. A. Traxler, J. Wu, K. M. Wannemacher, S. L. Cermignano, R. Voronov, S. L. Diamond, L. F. Brass, Hierarchical organization in the hemostatic response and its relationship to the plateletsignaling network, Blood 121 (2013) 1875-1885. 
[116] A. L. Fogelson, Y. H. Hussain, K. Leiderman, Blood clot formation under flow: The importance of factor xi depends strongly on platelet count, Biophys. J. 102 (2012) 10-18.

[117] A. Yazdani, H. Li, J. D. Humphrey, G. E. Karniadakis, A general sheardependent model for thrombus formation, PLOS Computational Biology 13 (1) (2017) e1005291.

[118] M. J. Maxwell, E. Westein, W. S. Nesbitt, S. Giuliano, S. M. Dopheide, S. P. Jackson, Identification of a 2-stage platelet aggregation process mediating shear-dependent thrombus formation, Blood 109 (2007) 566-576.

1835 [119] F. Storti, T. H. S. van Kempen, F. N. van de Vosse, A continuum model for platelet plug formation and growth, Int. J. Numer. Meth. Biomed. Eng. 30 (2014) 634-658.

[120] E. S. Babushkina, N. M. Bessonov, F. I. Ataullakhanov, M. A. Panteleev, Continuous modeling of arterial platelet thrombus formation using a spa-

[121] A. Tokarev, I. Sirakov, G. Panasenko, V. Volpert, E. Shnol, A. Butylin, F. Ataullakhanov, Continuous mathematical model of platelet thrombus formation in blood flow, Russian Journal of Numerical Analysis and Mathematical Modelling 27 (2) (2012) 192-212.

[122] J. Haga, Y.-S. Li, S. Chien, Molecular basis of the effects of mechanical stretch on vascular smooth muscle cells, J. Biomech. 40 (2007) 947-960. 
[123] J. Gonzalez-Ferrandez, B. Ermentrout, On the origin and dynamics of the vasomotion of small arteries, Math. Biosci. 119 (2007) 127-167.

[124] M. Koenigsberger, R. Sauser, J. Beny, J. Meister, Role of the endothelium on arterial vasomotion., Biophys. J. 88 (2005) 3845-3854.

[125] M. Koenigsberger, R. Sauser, J. Beny, J. Meister, Effects of arterial wall stress on vasomotion, Biophys. J. 91 (2006) 1663-1674.

[126] T. Takenaka, H. Suzuki, H. Okada, K. Hayashi, Y. Kanno, T. Saruta, Mechanosensitive cation channels mediate afferent arteriolar myogenic constriction in the isolated rat kidney, J. Physiol. 511 (1998) 245-253.

[127] T. David, S. Alzaidi, H. Farr, Coupled autoregulation models in the cerebro-vasculature, J. Eng. Math. 64 (2009) 403-415.

[128] M. H. Flamm, T. V. Colace, M. S. Chatterjee, H. Jing, S. Zhou, D. Jaeger, L. F. Brass, T. Sinno, S. L. Diamond, Multiscale prediction of patientspecific platelet function under flow, Blood 120 (2012) 190-198.

[129] D. Mori, K. Yano, K. Tsubota, T. Ishikawa, S. Wada, T. Yamaguchi, Simulation of platelet adhesion and aggregation regulated by fibrinogen and von willebrand factor, Thromb Haemost. 99 (2008) 108-115.

[130] N. A. Mody, M. R. King, Platelet adhesive dynamics. part i: characterization of platelet hydrodynamic collisions and wall effects, Biophys. J. 95 (2008) 2539-2555.

[131] N. A. Mody, M. R. King, Platelet adhesive dynamics. part ii: high shearinduced transient aggregation via gpiba-vwf-gpiba bridging, Biophys. J. 95 (2008) 2556-2574.

[132] C. Pozrikidis, Orbiting motion of a freely suspended spheroid near a plane wall, J. Fluid Mech. 541 (2005) 105-114.

[133] C. Pozrikidis, Flipping of an adherent blood platelet over a substrate., J Fluid Mech. 568 (2005) 161-172. 
[134] Z. Xu, N. Chen, S. C. Shadden, J. E. Marsden, M. M. Kamocka, E. D.

Rosen, M. Alber, Study of blood flow impact on growth of thrombi using a multiscale model, Soft Matter 5 (2009) 769-779.

[135] S. Succi, The Lattice Boltzmann Equation for Fluid Dynamics and Beyond (Numerical Mathematics and Scientific Computation), Oxford University Press, USA, 2001.

[136] D. Rodriguez, D. Einarsson, A. Carpio, Biofilm growth on rugose surfaces, Phys. Rev. E 86 (2012) 061914.

[137] F. Graner, J. A. Glazier, Simulation of biological cell sorting using a twodimensional extended potts model, Phys. Rev. Lett. 69 (1992) 2013-2016.

[138] V. Andasari, R. T. Roper, M. H. Swat, M. A. J. Chaplain, Integrating intracellular dynamics using compucell3d and bionetsolver: applications to multiscale modelling of cancer cell growth and invasion, PLoS ONE 7 (2012) e33726.

[139] M. Dennin, Discontinuous jamming transitions in soft materials: coexistence of flowing and jammed states, J. Phys.: Condens. Matter 20 (2008) 283103.

[140] S. W. Schneider, S. Nuschele, A. Wixforth, C. Gorzelanny, A. AlexanderKatz, R. R. Netz, M. F. Schneider, Shear-induced unfolding triggers adhesion of von willebrand factor fibers, Proc. Nat. Acad. Sci. 104 (2007) 7899-7903.

[141] N. Begent, G. V. R. Born, Growth rate in vivo of platelet thrombi, produced by iontophoresis of adp, as a function of mean blood flow velocity, Nature 227 (1970) 926-930.

[142] A. W. Tilles, E. C. Eckstein, The near-wall excess of platelet-sized particles in blood flow: its dependence on hematocrit and wall shear rate., Microvasc. Res. 33 (1987) 211-223. 
[143] B. Woldhuis, G. J. Tangelder, D. Slaaf, R. S. Reneman, Concentration profile of blood platelets differs in arterioles and venules, Am. J. Physiol. 262 (1992) H1217-H1223.

[144] W. Wang, N. A. Mody, M. R. King, Multiscale model of platelet translocation and collision, J Comput Phys. 244 (2013) 223-235.

[145] T. Skorczewski, L. C. Erickson, A. L. Fogelson, Platelet motion near a vessel wall or thrombus surface in two-dimensional whole blood simulations, Biophys. J. 104 (2013) 1764-1772.

[146] A. P. Spann, J. E. Campbell, S. R. Fitzgibbon, A. Rodriguez, A. P. Cap, L. H. Blackbourne, E. S. G. Shaqfeh, The effect of hematocrit on platelet adhesion: Experiments and simulations, Biophysical Journal 111 (2016) $577-588$.

[147] G. I. Bell, Models for the specific adhesion of cells to cells, Science 200 (1978) 618-627.

[148] E. Evans, D. Berk, A. Leung, Detachment of agglutinin-bonded red blood cells. i. forces to rupture molecular-point attachments, Biophysics Journal 59 (1991) 838-848.

[149] E. Evans, K. Ritchie, Dynamic strength of molecular adhesion bonds, Biophysics Journal 72 (1997) 1541-1555.

[150] G. Bell, M. Dembo, P. Bongrand, Cell adhesion. competition between nonspecific repulsion and specific bonding., Biophysics Journal 45 (1984) 1051-1064.

[151] J. Piper, R. Swerlick, C. Zhu, Determining force dependence of twodimensional receptor-ligand binding affnity by centrifugation, Biophysics Journal 74 (1998) 492-513.

[152] M. Dembo, D. Torney, K. Saxman, D. Hammer, The reaction-limited kinetics of membrane-to-surface adhesion and detachment, Proceedings of the Royal Society of London B 234 (1988) 55-83. 
[153] M. Dembo, On peeling an adherent cell from a surface, Lect. Math. Life Sci. 24 (1994) 51-77.

[154] W. Thomas, E. Trintchina, M. Forero, V. Vogel, E. Sokurenko, Bacterial adhesion to target cells enhanced by shear force, Cell 109 (7) (2002) 913923.

[155] B. Marshall, M. Long, J. Piper, T. Yago, R. McEver, C. Zhu, Direct observation of catch bonds involving cell-adhesion molecules, Nature 423 (6936) (2003) 190-193.

[156] Y. V. Pereverzev, O. V. Prezhdo, M. Forero, E. V. Sokurenko, W. E. Thomas, The two-pathway model for the catch-slip transition in biological adhesion, Biophysical Journal 89 (2005) 1446-1454.

${ }_{1940}$ [157] W. Thomas, M. Forero, O. Yakovenko, L. Nilsson, P. Vicini, E. Sokurenko, V. Vogel, Catch-bond model derived from allostery explains forceactivated bacterial adhesion, Biophys J. 90 (2006) 753-764.

[158] W. Thomas, Catch bonds in adhesion, Annu. Rev. Biomed. Eng. 10 (2008) $39-57$.

${ }_{1945}$ [159] T. Yago, J. Lou, T. Wu, J. Yang, J. J. Miner, L. Coburn, J. A. Lopez, M. A. Cruz, J.-F. Dong, L. V. McIntire, R. P. McEver, C. Zhu, Platelet glycoprotein ib $\alpha$ forms catch bonds with human wt vwf but not with type 2b von willebrand disease vwf, J. Clin. Invest. 118 (2008) 3195-3207.

[160] J. Kim, C.-Z. Zhang, X. Zhang, T. A. Springer, A mechanically stabilized receptor-ligand flex-bond important in the vasculature, Nature 466 (2010) 992-995.

[161] D. Hammer, S. Apte, Simulation of cell rolling and adhesion on surfaces in shear flow: general results and analysis of selectin-mediated neutrophil adhesion, Biophysics Journal 63 (1992) 35-57. 
[162] D. Hammer, D. Lauffenburger, A dynamical model for receptor-mediated cell adhesion to surfaces, Biophysics Journal 52 (1987) 475-487.

[163] E. F. Krasik, D. A. Hammer, A semianalytic model of leukocyte rolling, Biophysical Journal 87 (2004) 2919-2930.

[164] V. Barsegov, D. Thirumalai, Dynamics of unbinding of cell adhesion molecules: Transition from catch to slip bonds, Proc.Nat.Acad.Sci.USA 102 (6) (2005) 1835-1839.

[165] S. Reboux, G. Richardson, O. Jensen, Bond tilting and sliding friction in a model of cell adhesion, Proc. R. Soc. A 464 (2008) 447-467.

[166] S. Sircar, D. M. Bortz, Impact of flow on ligand-mediated bacterial flocculation, Mathematical Biosciences 245 (2013) 314-321.

[167] C. Aponte-Santamaria, V. Huck, S. Posch, A. K. Bronowska, S. Grässle, M. A. Brehm, T. Obser, R. Schneppenheim, P. Hinterdorfer, S. W. Schneider, C. Baldauf, F. Gräter, Force-sensitive autoinhibition of the von willebrand factor is mediated by interdomain interactions, Biophysical Journal 108 (2015) 2312-2321.

[168] D. Sankar, K. Hemalatha, A non-newtonian fluid flow model for blood flow through a catheterized artery-steady flow, Applied Mathematical Modelling 31 (9) (2007) 1847-1864.

[169] Y. C. Fung, Biomechanics: Circulation. Second edition., Springer-Verlag New York, Inc., 1997.

[170] S. R. Subia, M. S. Ingber, L. A. Mondy, S. A. Altobelli, A. L. Graham, Modelling of concentrated suspensions using a continuum constitutive equation, J. Fluid Mech. 373 (1998) 193-219.

[171] P. Goodman, E. Barlow, P.M.Crapo, S.F.Mohammad, K. Solen, Computational model of device-induced thrombosis and thromboembolism, Annals of Biomedical Engineering 33 (6) (2005) 780-797. 
[172] L. Mountrakis, E. Lorenz, A. G. Hoekstra, Scaling of shear-induced diffusion and clustering in a blood-like suspension, European Phys.Lett. 114 (2016) 14002.

[180] H. Chen, J. I. Angerer, M. Napoleone, A. J. Reininger, S. W. Schneider, A. Wixforth, M. F. Schneider, A. Alexander-Katz, Hematocrit and flow rate regulate the adhesion of platelets to von willebrand factor, Biomicrofluidics 7 (2013) 064113. 
[181] A. L. Fogelson, R. D. Guy, Immersed-boundary-type models of intravascular platelet aggregation, Computer Methods in Applied Mechanics and Engineering 197 (25-28) (2008) 2087-2104.

[182] D. Mori, K. Yano, K. Tsubota, T. Ishikawa, S. Wada, T. Yamaguchi, Computational study on effect of red blood cells on primary thrombus formation, Thrombosis Research 123 (2008) 114-121.

[183] G. Segre, A. Silberberg., Radial particle displacements in poiseuille flow of suspensions, Nature 189 (1961) 209-210.

[184] T. M. Geislinger, T. Franke, Hydrodynamic lift of vesicles and red blood cells in flow - from fahraeus \& lindqvist to microfluidic cell sorting, Advances in colloid and interface science 208 (2014) 161-176.

[185] K. Vahidkhah, P. Bagchi, Microparticle shape effects on margination, near- wall dynamics and adhesion in a three-dimensional simulation of red blood cell suspension, Soft Matter 11 (2015) 2097-109.

[186] L. M. Crowl, A. L. Fogelson, Computational model of whole blood exhibiting lateral platelet motion induced by red blood cells, Int. J. Numer. Method biomed. Eng. 26 (2010) 471-487.

[187] E. Evans, R. Skalak, Mechanics and thermodynamics of biomembranes., CRC Press Inc., Boca Raton., 1980.

[188] T. M. Fischer, C. W. M. Haest, M. StohrLiesen, H. Schmid-Schonbein, R. Skalak, The stress-free shape of the red blood cell membrane, Biophys. J. 34 (1981) 409-422.

[189] J. C. Hansen, R. Skalak, S. Chien, A. Hoger, An elastic network model based on the structure of the red blood cell membrane skeleton, Biophysical Journal 70 (1996) 146-166.

[190] J. C. Hansen, R. Skalak, S. Chien, A. Hoger, Influence of network topology on the elasticity of the red blood cell membrane skeleton, Biophysical Journal 72 (1997) 2369-2381. 
[191] I. Pivkin, G. Karniadakis, Accurate coarse-grained modeling of red blood cells, Phys. Rev. Lett. 101 (11) (2008) 118105.

[192] D. Reasor, J. Clausen, C. Aidun, Coupling the lattice-boltzmann and spectrin-link methods for the direct numerical simulation of cellular blood flow, Int. J. Numer. Methods Fluids 68 (6) (2012) 767-781.

[193] J. Li, M. Dao, C. Lim, S. Suresh, Spectrin-level modeling of the cytoskeleton and optical tweezers stretching of the erythrocyte, Biophys. J. 88 (5) (2005) 3707-3719.

[194] S. Suresh, J. Spatz, J. Mills, A. Micoulet, M. Dao, C. Lim, M. Bail, T. Seufferlein, Connections between single-cell biomechanics and human disease states: gastrointestinal cancer and malaria, Acta Biomaterialia 1 (2005) 15-30.

[195] D. Fedosov, B. Caswell, G. Karniadakis, Systematic coarse-graining of spectrin-level red blood cell models, Comput. Methods Appl. Mech. Eng. 199 (29-32) (2010) 1937-1948.

[196] M. Dao, C. Lim, S. Suresh, Mechanics of the human red blood cell deformed by optical tweezers, J. Mech. Phys. Solids. 51 (2003) 2259.

[197] J. P. Mills, L. Qie, M. Dao, C. T. Lim, S. Suresh, Nonlinear elastic and viscoelastic deformation of the human red blood cell with optical tweezers, Mol. Cell. Biomech. 1 (2004) 169.

[198] M. M. Dupin, I. Halliday, C. M. Care, L. Alboul, L. L. Munn, Modeling the flow of dense suspensions of deformable particles in three dimensions, Phys. Rev. E. 75 (2007) 066707.

[199] B. Kaoui, T. Krüger, J. Harting, How does confinement affect the dynamics of viscous vesicles and red blood cells?, Soft Matter 8 (2012) 9246-9252.

[200] L. Crowl, A. L. Fogelson, Analysis of mechanisms for platelet near-wall excess under arterial blood flow conditions, J. Fluid Mech. 676 (2010) $348-375$. 
[201] L. Mountrakis, E. Lorenz, O. Malaspinas, S. Alowayyed, B. Chopard, A. G. Hoekstra, Parallel performance of an ib-lbm suspension simulation framework, Journal of Computational Science 9 (2015) 45-50.

[202] C. Peskin, The immersed boundary method, Acta Numer. 11 (1) (2002) $479-517$.

[203] Z. Guo, C. Zheng, B. Shi, Discrete lattice effects on the forcing term in the lattice boltzmann method, Phys. Rev. E. 65 (2002) 046308.

[204] A. Leonardi, F. K. Wittel, M. Mendoza, H. J. Herrmann, Coupled dem$\mathrm{lbm}$ method for the free-surface simulation of heterogeneous suspensions, Computational Particle Mechanics 1 (1) (2014) 3-13.

[205] I. V. Pivkin, P. D. Richardson, G. Karniadakis, Blood flow velocity effects and role of activation delay time on growth and form of platelet thrombi, Proc. Nat. Acad. Sci. 103 (46) (2006) 17164-17169.

[206] P. Koumoutsakos, Multiscale flow simulations using particles, Annu Rev Fluid Mech 37 (2005) 457-487.

[207] D. Alizadehrad, Y. Imai, K. Nakaaki, T. Ishikawa, T. Yamaguchi, Quantification of red blood cell deformation at high-hematocrit blood flow in microvessels, Journal of Biomechanics 45 (2012) 2684-2689.

[208] Y. Imai, H. Kondo, T. Ishikawa, T. Yamaguchi, Modeling of hemodynamics arising from malaria infection, Journal of Biomechanics 43 (2010) 1386-1393.

[209] P. Espanol, M. Revenga, Smoothed dissipative particle dynamics, Phys. Rev. E: Stat., Nonlinear, SoftMatter Phys. 67 (2003) 026705.

[210] D. A. Fedosov, G. Gompper, White blood cell margination in microcirculation., Soft Matter 10 (17) (2014) 2961-70.

[211] C. Pozrikidis, Boundary Integral and Singularity Methods for Linearized Viscous Flow, Cambridge University Press, Cambridge, UK, 1992. 
[212] A. Raue, M. Schilling, J. Bachmann, A. Matteson, M. Schelke, D. Kaschek, S. Hug, C. Kreutz, B. Harms, F. Theis, U. Klingmüller,

[217] M. J. Fasco, L. M. Principe, W. A. Walsh, P. A. Friedman, Warfarin inhibition of vitamin $\mathrm{k} \mathrm{2}$, 3-epoxide reductase in rat liver microsomes, Biochemistry 22 (24) (1983) 5655-5660.

[218] Warfarin INR targets, http://www.globalrph.com/warfarin_inr_ targets.htm. Accessed: 2016-02-22. 